\title{
Rigid supersymmetric backgrounds of 3-dimensional Newton-Cartan supergravity
}

\section{Gino Knodel, Pedro Lisbão and James T. Liu}

Michigan Center for Theoretical Physics, Randall Laboratory of Physics, The University of Michigan, Ann Arbor, MI 48109-1040, U.S.A.

E-mail: gknodel@umich.edu, plisbao@umich.edu, jimliu@umich.edu

ABSTRACT: Recently, a non-relativistic off-shell formulation of three dimensional NewtonCartan supergravity was proposed as the $c \rightarrow \infty$ limit of three dimensional $\mathcal{N}=2$ supergravity [1]. In the present paper we study supersymmetric backgrounds within this theory. Using integrability constraints for the non-relativistic Killing spinor equations, we explicitly construct all maximally supersymmetric solutions, which admit four supercharges. In addition to these solutions, there are $\frac{1}{2}$-BPS solutions with reduced supersymmetry. We give explicit examples of such backgrounds and derive necessary conditions for backgrounds preserving two supercharges. Finally, we address how supersymmetric backgrounds of $\mathcal{N}=2$ supergravity are connected to the solutions found here in the $c \rightarrow \infty$ limit.

KEYwORDS: Holography and condensed matter physics (AdS/CMT), Supergravity Models

ARXiv EPrint: 1512.04961 


\section{Contents}

1 Introduction $\quad 2$

2 Newton-Cartan gravity 4

2.1 Vielbein formalism 5

2.2 Constraints and adapted coordinates 6

2.3 Connection and interpretation of $m_{\mu} \quad 7$

3 Off-shell Newton-Cartan supergravity 4

4 Non-relativistic supersymmetric backgrounds $\quad 9$

$\begin{array}{lll}4.1 & \text { Maximally supersymmetric solutions } & 10\end{array}$

$\begin{array}{lll}\text { 4.1.1 Connection coefficients } & 12\end{array}$

$\begin{array}{ll}\text { 4.1.2 Killing spinors } & 13\end{array}$

$4.2 \frac{1}{2}$-BPS solutions 14

4.2.1 Backgrounds with two supercharges of the form $\left(0, \epsilon_{-}\right) \quad 14$

$\begin{array}{ll}\text { 4.2.2 Backgrounds with two supercharges of the form }\left(\epsilon_{+}, 0\right) & 15\end{array}$

4.2.3 Backgrounds with two supercharges of the form $\left(\epsilon_{+}, F \epsilon_{+}\right) \quad 16$

$\begin{array}{ll}\text { 4.2.4 A nontrivial example } & 17\end{array}$

5 Rigid backgrounds of $\mathcal{N}=2$ supergravity 18

5.1 The $f_{\mu}=0$ case 20

$\begin{array}{lll}5.2 & \text { The timelike case } & 20\end{array}$

5.3 The spacelike case 21

5.4 The null case 22

6 Non-relativistic limit of $\mathcal{N}=2$ supergravity 23

$\begin{array}{ll}6.1 & \text { Killing spinor equation } \\ \end{array}$

$\begin{array}{lll}7 & \text { Discussion } & 26\end{array}$

$\begin{array}{lr}\text { A Notation and conventions } & 27\end{array}$

B Bilinear analysis of $\mathcal{N}=2$ backgrounds $\quad 28$

$\begin{array}{ll}\text { B.1 Timelike case } & 29\end{array}$

B.2 Null case 31 


\section{Introduction}

The study of non-relativistic field theories and their holographic duals has led to a renewed recent interest in Newton-Cartan gravity [2-12]. The latter theory is formulated as a covariant description of Newtonian gravity, incorporating the notion of absolute time in a geometric framework (see e.g. [13, 14] for pedagogical introductions). It has been argued that in the context of non-relativistic holography, Newton-Cartan gravity is the natural geometric language in which the bulk-boundary dictionary is to be developed. For example, the boundary geometry of Lifshitz spacetime has been shown to be described by a NewtonCartan geometry with torsion $[5,6]$. On the other hand, starting with a non-relativistic field theory, Newton-Cartan gravity arises as a means of introducing (non-relativistic) coordinate invariance: while a (relativistic) CFT may be coupled to dynamical gravity by introducing a metric $g_{\mu \nu}$ with dynamics governed by General Relativity, non-relativistic field theories couple naturally to Newton-Cartan gravity, which can be formulated in terms of two degenerate metrics, $\tau_{\mu \nu}$ and $h^{\mu \nu}$. This insight has been used to construct effective field theories for quantum Hall states and study universal features of the theories obtained in this way [2-4].

Although the degrees of freedom in Newton-Cartan gravity differ fundamentally from those of General Relativity, many conceptual aspects still carry forward to the nonrelativistic case. In the same way that General Relativity can be written as a gauge theory of the Poincaré algebra, Newton-Cartan gravity can be formulated as a gauge theory of the Bargmann algebra, which is the centrally extended Galilei algebra [15]. The formulation of gravity as a gauge theory has the advantage that introducing supersymmetry to construct theories of supergravity is relatively straightforward. In complete analogy to the case of conventional (relativistic) supergravity, it is therefore possible to construct supergravity theories with a non-relativistic supersymmetry group. In three dimensions, an on-shell theory of Newton-Cartan supergravity with four real supercharges was constructed using a vielbein approach in [16]. Moreover, by using a non-relativistic limiting procedure, the authors of [1] were able to construct an off-shell version of the latter theory, starting from off-shell $\mathcal{N}=2$ supergravity $[17,18]$. These recent developments allow us to ask many of the interesting questions that arise within the context of supersymmetry and supergravity, applied to a non-relativistic context.

The main motivation for this paper is the prospect of using Newton-Cartan supergravity to elucidate some open questions in non-relativistic gauge/gravity dualities. In the standard case of relativistic AdS/CFT, much recent progress in understanding various dualities has been made by using supersymmetric localization. This technique allows one to calculate observables in supersymmetric theories exactly, without having to resort to perturbation theory (for a review of recent progress see [19] and references therein). The new results obtained this way can be used to provide precision tests of AdS/CFT: for example, the free energy of $\mathcal{N}=2^{\star}$ theories on $S^{4}$, calculated via localization [20] matches the result that one obtains from a holographic calculation [21]. Given that in the context of non-relativistic holography, a microscopic description in terms of branes is not always available to motivate the duality between non-relativistic field theories and gravity, it would be desirable to find similar precision tests for non-relativistic AdS/CFT. 
Holographic results for observables in non-relativistic geometries such as Lifshitz and Schrödinger spacetimes are plentiful. However, on the field theory side exact results are naturally difficult to obtain, due to the strongly coupled nature of the theories involved. Given the success of studying supersymmetric theories in the relativistic case, in particular using localization, one concrete open question is: is there a non-relativistic analog of supersymmetric localization? To answer this question, it is first necessary to understand and further explore the notion of "non-relativistic supersymmetry" itself. While specific examples of non-relativistic supersymmetric field theories have been constructed previously [22-24], many aspects of this subject still remain unexplored.

One interesting general question is which backgrounds of Newton-Cartan gravity admit non-relativistic supersymmetry, and how to systematically construct Lagrangians on these backgrounds. In the relativistic case, a systematic approach to this question was outlined by Festuccia and Seiberg [25]. Starting with an off-shell formulation of supergravity coupled to matter fields, one proceeds to take the "rigid limit" by freezing out graviton and gravitino fluctuations, thereby obtaining a supersymmetric theory on a curved background. The conditions for a background to be supersymmetric are found by demanding that the gravitino variation vanishes. This in turn leads to Killing spinor equations in curved space, which can be studied systematically to classify supersymmetric backgrounds [26-31].

In this paper, we initiate a similar approach to classifying curved Newton-Cartan backgrounds that admit field theories with non-relativistic supersymmetry. Starting with the off-shell version of three-dimensional Newton-Cartan supergravity found in [1], we proceed to decouple gravity. Demanding that the gravitino and its variation vanish leads to a non-relativistic Killing spinor equation, which we analyze in detail. Using integrability conditions, we can derive the necessary and sufficient conditions for backgrounds to admit four supercharges (unbroken supersymmetry), and also study examples of backgrounds with reduced supersymmetry ( $\frac{1}{2}$-BPS solutions). The supersymmetric solutions found this way can be characterized by a "gravitational force" field $\Phi_{i}(t, \vec{x})=\Gamma_{00}^{i}$, and a "Coriolis force" field $C(t, \vec{x})=\frac{1}{2} \epsilon_{i j} \Gamma_{0 j}^{i}$, both of which represent the curvature induced by foliating the temporal slices in a non-trivial way along the absolute time direction $\tau_{\mu}$. Interestingly, a necessary condition for a background to preserve any number of supersymmetry is that the spatial curvature, captured by $\Gamma_{j k}^{i}$, vanishes.

Since backgrounds of Newton-Cartan gravity are formulated in a somewhat unfamiliar language, using either two degenerate metrics, or one spatial metric and a "velocity" field $\tau_{\mu}$, it is instructive to connect our results to those for the relativistic $\mathcal{N}=2$ supergravity theory. Given that the Newton-Cartan supergravity theory of [1] was obtained as the $c \rightarrow \infty$ limit of the relativistic theory [17, 18], one may ask whether the same limit can be taken already at the level of the Killing spinor equations themselves, in order to relate relativistic to non-relativistic backgrounds. Although taking this limit is possible, we are not guaranteed to end up with the same BPS conditions for non-relativistic backgrounds that we do by starting with Newton-Cartan supergravity, and freezing out gravity (see figure 1). In other words, the rigid limit and the $c \rightarrow \infty$ limit do not commute. The non-commutativity is due to the additional constraints on auxiliary fields that are imposed in Newton-Cartan supergravity, where the gravitino is generally nonzero. These conditions are not needed at the level of rigid supersymmetry without gravity. 


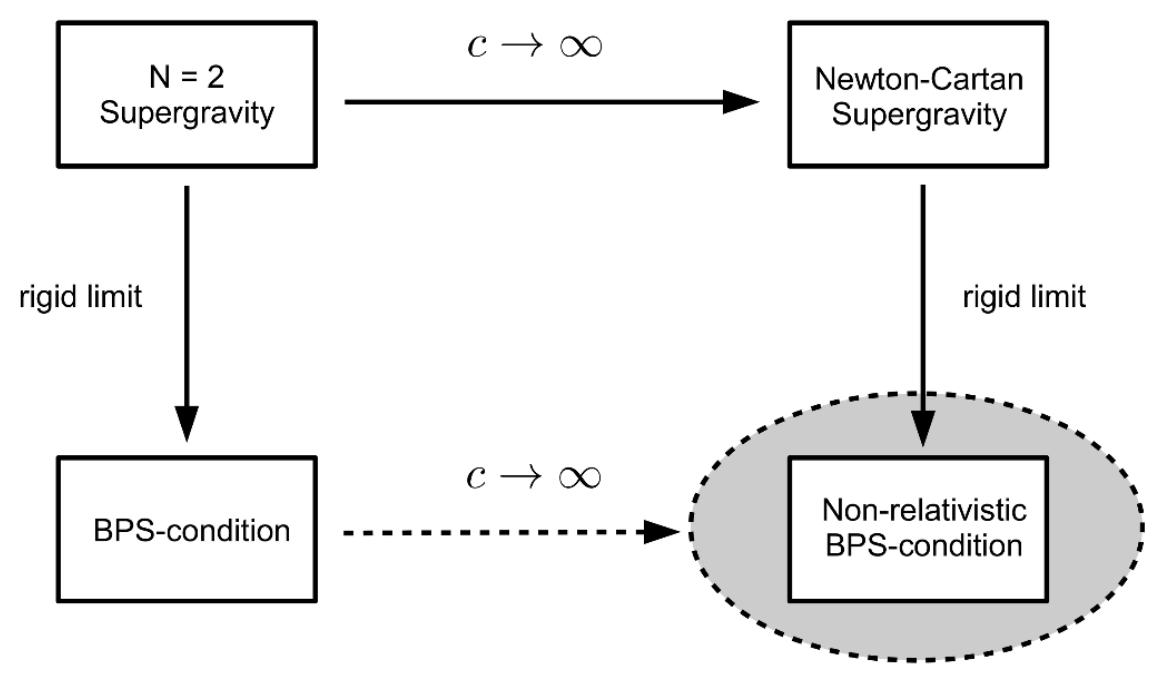

Figure 1. The difference between the non-relativistic BPS-condition, (5.5) through (5.8), obtained by taking the rigid limit of Newton-Cartan supergravity, and the set of backgrounds obtained by taking the non-relativistic limit of the relativistic supersymmetric solutions (grey). In general, the latter is a superset of the former.

The rest of this paper is organized as follows. In section 2, we briefly review NewtonCartan gravity in both the metric and vielbein formalism. In section 3, we review the off-shell version of three-dimensional Newton-Cartan supergravity found in [1], and in particular the limiting procedure that was used to derive the theory. In section 4, we derive and analyze the non-relativistic Killing spinor equation. Using integrability conditions, we determine all backgrounds with maximal supersymmetry, and also study examples of backgrounds with reduced supersymmetry. In section 5 , we turn to the relativistic $\mathcal{N}=2$ supergravity theory and study supersymmetric backgrounds using the same method of rigid supersymmetry as before. Using integrability, we derive all solutions admitting four relativistic supercharges. In section 6 , we discuss the $c \rightarrow \infty$ limit of the relativistic Killing spinor equation, and show that it leads to a bigger class of non-relativistic solutions than those found in section 4 . We conclude with a discussion of our results and point towards some interesting future directions.

\section{Newton-Cartan gravity}

To set the stage for our supergravity analysis, let us first review Newton-Cartan gravity [13, 14]. Newton-Cartan gravity is a covariant formulation of Newtonian gravity. Due to its non-relativistic nature, this theory is commonly formulated in terms of a temporal metric $\tau_{\mu \nu}$, and a separate spatial metric $h^{\mu \nu}$, making spatial and temporal distances two separate, well-defined quantities. Both metrics are degenerate, which can be understood heuristically by considering the example of the non-relativistic limit of the Minkowski metric [15]:

$$
\eta_{\mu \nu}=\left(\begin{array}{cc}
-c^{2} & 0 \\
0 & I_{D-1}
\end{array}\right), \quad \eta^{\mu \nu}=\left(\begin{array}{cc}
-1 / c^{2} & 0 \\
0 & I_{D-1}
\end{array}\right)
$$


In the limit $c \rightarrow \infty$, the metric naturally splits into a temporal and a spatial metric:

$$
\eta_{\mu \nu} \rightarrow \tau_{\mu \nu}, \quad \eta^{\mu \nu} \rightarrow h^{\mu \nu},
$$

where in our case, $\tau_{\mu \nu}=-c^{2} \delta_{\mu}^{0} \delta_{\nu}^{0}, h^{\mu \nu}=\delta^{i j}$. The degeneracy can be expressed as

$$
\tau_{\mu \rho} h^{\rho \nu}=0
$$

The temporal metric may be written as $\tau_{\mu \nu}=\tau_{\mu} \tau_{\nu}$. Intuitively, we can understand the geometries within Newton-Cartan gravity in the following way: the 1-form $\tau_{\mu}$ defines a global time direction. At each moment in time, there is a Riemannian space with (inverse) metric $h^{\mu \nu}$. The connection to Newtonian gravity is established by choosing the curvature of the resulting manifold in such a way that the geodesics of particles moving in the curved space geometry are equivalent to the curved paths of classical particles in flat space.

\subsection{Vielbein formalism}

In this paper, we will also use an alternative formulation of Newton-Cartan gravity in terms of vielbein fields [15]. Recall that General Relativity in $D$ dimensions can be formulated as a gauge theory of the Poincaré algebra, which has generators $P_{A}$ and $M_{A B}(A, B=$ $0,1, \ldots, D-1)$. The associated gauge fields are the vielbein $E_{\mu}^{A}$, and the spin connection $\Omega_{\mu}^{A B}$. Newton-Cartan gravity can be written in the same language. To accomplish this, we first identify the generators of the non-relativistic symmetry group. In our case, the generators are given by time translations $H$, spatial translations $P_{a}$, rotations $J_{a b}$, and Galilean boosts $G_{a}(a=1, \ldots, D-1)$, which together form the Galilean algebra. To connect the relativistic and non-relativistic symmetry groups, it turns out to be more natural to consider the Bargmann algebra, which is the central extension of the Galilean algebra by a U(1) generator $Z$ [15]. The Bargmann algebra can be obtained by performing an Inönü-Wigner contraction of the Poincaré algebra [1]. For each of the generators, we then introduce corresponding gauge fields:

$$
\begin{aligned}
\text { time translations: } & H \leftrightarrow \tau_{\mu} \\
\text { spatial translations: } & P_{a} \leftrightarrow e_{\mu}^{a} \\
\text { rotations: } & J_{a b} \leftrightarrow \omega_{\mu}^{a b} \\
\text { Galilean boosts: } & G_{a} \leftrightarrow \omega_{\mu}^{a} \\
\mathrm{U}(1): & Z \leftrightarrow m_{\mu}
\end{aligned}
$$

We see that the spacetime-translation generator $P_{A}$ of the Poincaré algebra has split up into time translations $H$ and spatial translations $P_{a}$. Correspondingly, the vielbein splits as $E_{\mu}^{A} \rightarrow\left(\tau_{\mu}, e_{\mu}^{a}\right)$, where $\tau_{\mu}$ is a "temporal vielbein" and $e_{\mu}^{a}$ is a "spatial vielbein". In a similar fashion, the spin connection $\Omega_{\mu}^{A B}$ splits up into an $\mathrm{SO}(2)$ spin-connection $\omega_{\mu}^{a b}$, and a boost connection $\omega_{\mu}^{a}$. Finally, the abelian gauge field $Z$ provides a central extension of the Galilean algebra (2.4)-(2.7) to the full Bargmann algebra. It is needed to consistently perform the contraction of the Poincare algebra. Later we will see that the geometric role of 
the corresponding gauge field $m_{\mu}$ is to define the rules of parallel transport, or equivalently to define a connection $\Gamma$.

Next, we define inverse vielbein fields $e_{a}^{\mu}, \tau^{\mu}$ such that

$$
\begin{array}{rlrl}
e_{a}^{\mu} e_{\mu}^{b} & =\delta_{a}^{b}, & \tau^{\mu} \tau_{\mu}=1, \\
e_{a}^{\rho} e_{\mu}^{a}=\delta_{\mu}^{\rho}-\tau_{\mu} \tau^{\rho}, & \tau^{\mu} e_{\mu}^{a}=\tau_{\mu} e_{a}^{\mu}=0 .
\end{array}
$$

Note that flat spatial indices $a, b, \ldots$ are contracted using $\delta^{a b}$. The degenerate metrics introduced previously are given in terms of the vielbeine in the usual way:

$$
h_{\mu \nu}=e_{\mu}^{a} e_{\nu a}, \quad h^{\mu \nu}=e_{a}^{\mu} e^{\nu a}, \quad \tau_{\mu \nu}=\tau_{\mu} \tau_{\nu} .
$$

The constraints (2.10) then imply

$$
h^{\mu \nu} h_{\nu \rho}=\delta_{\mu}^{\rho}-\tau_{\mu} \tau^{\rho}, \quad h_{\mu \nu} \tau^{\nu}=h^{\mu \nu} \tau_{\nu}=0 .
$$

We will make use of these conditions when constructing explicit background metrics later. Although the vielbein formalism is useful to construct Newton-Cartan (super)gravity, we will also use the metric $h^{\mu \nu}$ when studying particular backgrounds, as it connects more directly to the familiar metric formulation of General Relativity.

\subsection{Constraints and adapted coordinates}

We can define gauge covariant curvatures of each of the gauge fields. In the relativistic (Poincaré) case, those curvatures are given by

$$
\begin{aligned}
R_{\mu \nu}{ }^{A}(E) & =2 \partial_{[\mu} E_{\nu]}^{A}-2 \Omega_{[\mu}^{A B} E_{\nu] B}, \\
R_{\mu \nu}{ }^{A B}(\Omega) & =2 \partial_{[\mu} \Omega_{\nu]}^{A B}-2 \Omega_{[\mu}^{A C} \Omega_{\nu] C}{ }^{B} .
\end{aligned}
$$

By imposing the first structure equation

$$
R_{\mu \nu}{ }^{A}(E)=0,
$$

we can solve for the spin connection $\Omega_{\mu}^{A B}$ in terms of $E_{\mu}^{A}$. In complete analogy with the relativistic case, we can define gauge covariant curvatures corresponding to each of the generators in Newton-Cartan gravity:

$$
\begin{aligned}
R_{\mu \nu}(H) & =2 \partial_{[\mu} \tau_{\nu]}, \\
R_{\mu \nu}^{a}(P) & =2 \partial_{[\mu} e_{\nu]}^{a}-2 \omega_{[\mu}^{a b} e_{\nu] b}-2 \omega_{[\mu}^{a} \tau_{\nu]}, \\
R_{\mu \nu}^{a b}(J) & =2 \partial_{[\mu} \omega_{\nu]}^{a b}-2 \omega_{[\mu}^{a c} \omega_{\nu] c}^{b} \\
R_{\mu \nu}{ }^{a}(G) & =2 \partial_{[\mu} \omega_{\nu]}^{a}-2 \omega_{[\mu}^{a b} \omega_{\nu] b} \\
R_{\mu \nu}(Z) & =2 \partial_{[\mu} m_{\nu]}-2 \omega_{[\mu}^{a} e_{\nu] a} .
\end{aligned}
$$

In the absence of additional structure, one can show that taking the non-relativistic limit of the torsion-free condition (2.15) consistently requires imposing the following constraints on the non-relativistic curvature tensors [1]:

$$
R_{\mu \nu}(H)=R_{\mu \nu}{ }^{a}(P)=R_{\mu \nu}(Z)=0 .
$$


These constraints can be used to determine the connections $\omega_{\mu}^{a b}$ and $\omega_{\mu}^{a}$ in terms of $\left(\tau_{\mu}, e_{\mu}^{a}, m_{\mu}\right)$ :

$$
\begin{aligned}
\omega_{\mu}^{a b} & =-2 e^{\nu[a} \partial_{[\mu} e_{\nu]}^{b]}+e_{\mu}^{c} e^{\rho a} e^{\nu b} \partial_{[\rho} e_{\nu]}^{c}-\tau_{\mu} e^{\rho a} e^{\nu b} \partial_{[\rho} m_{\nu]}, \\
\omega_{\mu}^{a} & =\tau^{\nu} \partial_{[\mu} e_{\nu]}^{a}+e_{\mu}^{b} e^{\rho a} \tau^{\nu} \partial_{[\rho} e_{\nu]}^{b}+e^{\nu a} \partial_{[\mu} m_{\nu]}-\tau_{\mu} e^{\rho a} \tau^{\nu} \partial_{[\rho} m_{\nu]} .
\end{aligned}
$$

A background of Newton-Cartan gravity is therefore uniquely determined by choosing $\left(\tau_{\mu}, e_{\mu}^{a}, m_{\mu}\right)$.

The constraint $R_{\mu \nu}(H)=2 \partial_{[\mu} \tau_{\nu]}=0$ gives rise to torsionless Newton-Cartan gravity. In fact, this torsion-free condition can be relaxed by including a background gauge field while taking the non-relativistic limit [32]. For our present analysis, however, we restrict to the torsionless theory, in which case we can locally write $\tau_{\mu}=\partial_{\mu} T\left(x^{\nu}\right)$. By construction, $\tau_{\mu}$ singles out a time-direction, so it is useful to introduce "adapted coordinates" by letting $T\left(x^{\mu}\right)=x^{0} \equiv t$, so that $\tau_{\mu}=\delta_{\mu}^{0}$. In these coordinates, the constraints (2.9) and (2.12) imply

$$
\begin{aligned}
\tau_{\mu} & =\delta_{\mu}^{0}, \\
\tau^{\mu} & =\left(1, v^{i}\right), \\
h^{\mu 0} & =0, \\
h_{\mu 0} & =-h_{\mu i} v^{i} .
\end{aligned}
$$

Note that our gauge choice does not completely fix the coordinates. In fact, there is a residual gauge freedom given by

$$
\begin{aligned}
t & \rightarrow t+\text { const. } \\
x^{i} & \rightarrow F^{i}\left(t, x^{j}\right),
\end{aligned}
$$

with $\operatorname{det} \frac{\partial F^{i}}{\partial x^{j}} \neq 0$.

\subsection{Connection and interpretation of $m_{\mu}$}

Given a background characterized by $\left(\tau_{\mu}, e_{\mu}^{a}, m_{\mu}\right)$, we can uniquely define a connection by imposing the vielbein postulate [15]

$$
\begin{aligned}
\partial_{\mu} e_{\nu}^{a}-\omega_{\mu}^{a b} e_{\nu b}-\omega_{\mu}^{a} \tau_{\nu}-\Gamma_{\nu \mu}^{\rho} e_{\rho}^{a} & =0 \\
\partial_{\mu} \tau_{\nu}-\Gamma_{\nu \mu}^{\lambda}{ }_{\nu} \tau_{\lambda} & =0
\end{aligned}
$$

which can be solved to find the connection coefficients

$$
\begin{aligned}
\Gamma_{\mu \nu}^{\rho} & =\tau^{\rho} \partial_{(\mu} \tau_{\nu)}+e_{a}^{\rho}\left(\partial_{(\mu} e_{\nu)}^{a}-\omega_{(\mu}^{a b} e_{\nu) b}-\omega_{(\mu}^{a} \tau_{\nu)}\right) \\
& =\tau^{\rho} \partial_{(\mu} \tau_{\nu)}+\frac{1}{2} h^{\rho \lambda}\left(\partial_{\nu} h_{\lambda \mu}+\partial_{\mu} h_{\lambda \nu}-\partial_{\lambda} h_{\mu \nu}+2 K_{\lambda(\mu} \tau_{\nu)}\right),
\end{aligned}
$$

where

$$
K_{\mu \nu}=2 \partial_{[\mu} m_{\nu]}
$$

As we will see, the definition of $\Gamma$ is the only place where $m_{\mu}$ shows up. Therefore, $m_{\mu}$ plays the role of determining the rules of parallel transport in a given background $\left(\tau_{\mu}, e_{\mu}^{a}\right)$. 
The Riemann tensor can be written in terms of the connection in the usual way:

$$
R_{\nu \rho \sigma}^{\mu}(\Gamma)=\partial_{\rho} \Gamma_{\nu \sigma}^{\mu}-\partial_{\sigma} \Gamma_{\nu \rho}^{\mu}+\Gamma_{\nu \sigma}^{\lambda} \Gamma_{\lambda \rho}^{\mu}-\Gamma_{\nu \rho}^{\lambda} \Gamma_{\lambda \sigma}^{\mu}
$$

Alternatively, we can express it in terms of the boost- and spin-curvature tensors of the Bargmann algebra:

$$
R_{\nu \rho \sigma}^{\mu}(\Gamma)=-e_{a}^{\mu}\left(R_{\rho \sigma}^{a}(G) \tau_{\nu}+R_{\rho \sigma}^{a b}(J) e_{\nu b}\right)
$$

\section{Off-shell Newton-Cartan supergravity}

Three-dimensional Newton-Cartan supergravity can be constructed as a gauge theory of the supersymmetric extension of the Bargmann algebra introduced in section 2.1 [16]. To derive an off-shell version of this theory, one starts with an off-shell realization of $\mathcal{N}=2$ supergravity and performs an Inönü-Wigner contraction that reduces the relativistic supersymmetry algebra to the super-Bargmann algebra [1].

In three dimensions, there are two inequivalent formulations of $\mathcal{N}=2$ supergravity, namely the $\mathcal{N}=(1,1)$ theory $[17,33,34]$ and the $\mathcal{N}=(2,0)$ theory [17]. We will focus on the $(2,0)$ theory, since it was used as a starting point for constructing the torsionless Newton-Cartan supergravity theory of [1]. The gravity multiplet of both $\mathcal{N}=2$ supergravity theories contains a vielbein $E_{\mu}^{A}(A=0,1,2)$ and two gravitini $\Psi_{\mu i}(i=1,2)$, which are Majorana spinors with two real components each. The off-shell multiplet of the $(2,0)$ theory additionally contains two gauge fields $M_{\mu}$ and $V_{\mu}$, as well as a scalar $D$. The variations of each field under supersymmetry can be found in $[1,17]$. Here we focus only on the transformation properties of the gravitino. Under a combined supersymmetry transformation (parametrized by two Majorana spinors $\eta_{i}$ ) and $\mathrm{U}(1)_{R}$-transformation (parametrized by $\rho$ ), the gravitino transforms as

$$
\delta \Psi_{\mu i}=\nabla_{\mu} \eta_{i}+\epsilon^{i j} \eta_{j} V_{\mu}-\gamma_{\mu} \eta_{i} D+\frac{1}{4} \gamma_{\mu} \gamma_{\rho \sigma} \hat{F}^{\rho \sigma} \epsilon^{i j} \eta_{j}-\epsilon^{i j} \Psi_{\mu j} \rho,
$$

where

$$
\hat{F}_{\mu \nu}=2 \partial_{[\mu} M_{\nu]}-\frac{1}{2} \epsilon^{i j} \bar{\Psi}_{[\mu i} \Psi_{\nu] j}
$$

and $\nabla_{\mu}=\partial_{\mu}-\frac{1}{4} \Omega_{\mu}^{A B} \gamma_{A B}$

Off-shell Newton-Cartan supergravity is constructed by taking a non-relativistic limit of the fields that mirrors the limit taken in the contraction of the Poincare algebra. Let us give a brief review of this limiting procedure, as outlined in [1]. One starts by redefining the bosonic fields as follows:

$$
\begin{aligned}
E_{\mu}^{A} & =\delta_{0}^{A}\left(\omega \tau_{\mu}+\frac{1}{2 \omega} m_{\mu}\right)+\delta_{a}^{A} e_{\mu}^{a}, \\
M_{\mu} & =\omega \tau_{\mu}-\frac{1}{2 \omega} m_{\mu}, \\
D & =\frac{1}{\omega} S
\end{aligned}
$$


where $a=1,2$. The spinors are first rewritten as

$$
\Psi_{ \pm}=\frac{1}{\sqrt{2}}\left(\Psi_{1} \pm \gamma_{0} \Psi_{2}\right), \quad \eta_{ \pm}=\frac{1}{\sqrt{2}}\left(\eta_{1} \pm \gamma_{0} \eta_{2}\right)
$$

and then rescaled according to

$$
\begin{array}{ll}
\Psi_{+}=\sqrt{\omega} \psi_{+}, & \eta_{+}=\sqrt{\omega} \epsilon_{+}, \\
\Psi_{-}=\frac{1}{\sqrt{\omega}} \psi_{-}, & \eta_{-}=\frac{1}{\sqrt{\omega}} \epsilon_{-} .
\end{array}
$$

Finally, the curvature form splits into spatial and temporal components as follows:

$$
\begin{aligned}
& \Omega_{\mu}^{a b}=\omega_{\mu}^{a b}+O\left(\frac{1}{\omega^{2}}\right), \\
& \Omega_{\mu}^{0 a}=\frac{1}{\omega} \omega_{\mu}^{a}+O\left(\frac{1}{\omega^{3}}\right) .
\end{aligned}
$$

The one-form $\omega_{\mu}^{a}$ is a boost connection, while $\omega_{\mu}^{a b}$ is a spin connection for spatial rotations.

Next, we take the limit $\omega \rightarrow \infty$, which can be thought of as taking $c \rightarrow \infty$. To eliminate divergences that appear in the transformation laws, one is forced to impose the following constraints on the bosonic fields:

$$
\begin{aligned}
\partial_{[\mu} \tau_{\nu]} & =0, \\
V_{\mu} & =-2 \tau_{\mu} S, \\
\hat{F}_{\mu \nu} & =2 \partial_{[\mu} M_{\nu]}-\frac{1}{2} \epsilon^{i j} \bar{\Psi}_{[\mu i} \Psi_{\nu] j}=0 .
\end{aligned}
$$

Finally, the non-relativistic variations of the gravitini take the form

$$
\begin{aligned}
& \delta \psi_{\mu+}=D_{\mu} \epsilon_{+}+S \tau_{\mu} \gamma_{0} \epsilon_{+}+\gamma_{0} \psi_{\mu+} \rho \\
& \delta \psi_{\mu-}=D_{\mu} \epsilon_{-}-3 S \tau_{\mu} \gamma_{0} \epsilon_{-}+\frac{1}{2} \omega_{\mu}^{a} \gamma_{a 0} \epsilon_{+}-S e_{\mu}^{a} \gamma_{a} \epsilon_{+}-\gamma_{0} \psi_{\mu-} \rho,
\end{aligned}
$$

where the derivative operator $D_{\mu} \equiv \partial_{\mu}-\frac{1}{4} \omega_{\mu}^{a b} \gamma_{a b}$ is covariant under local spatial rotations. The non-relativistic supergravity multiplet consists of $\tau_{\mu}, e_{\mu}^{a}, \psi_{\mu \pm}$, as well as the auxiliary fields $m_{\mu}$ and $S$. The vielbein $E_{\mu}^{A}$ has split up into a temporal vielbein $\tau_{\mu}$ and a separate spatial vielbein $e_{\mu}^{a}$ (see section 2.1). Finally, note that this construction gives rise to torsionless Newton-Cartan supergravity. The constraints (3.8), (3.9) and (3.10) can be lifted by instead considering the torsionful theory [35].

\section{Non-relativistic supersymmetric backgrounds}

To find backgrounds that respect non-relativistic supersymmetry, we proceed by demanding that the gravitini $\psi_{ \pm}$and their variations (3.11) and (3.12) vanish. This guarantees that the 
bosonic fields do not vary under supersymmetry, and in addition gives rise to the following Killing spinor equations:

$$
\begin{aligned}
& D_{\mu} \epsilon_{+}=-S \tau_{\mu} \gamma_{0} \epsilon_{+} \\
& D_{\mu} \epsilon_{-}=3 S \tau_{\mu} \gamma_{0} \epsilon_{-}-\frac{1}{2} \omega_{\mu}^{a} \gamma_{a 0} \epsilon_{+}+S e_{\mu}^{a} \gamma_{a} \epsilon_{+} .
\end{aligned}
$$

Each solution $\left(\epsilon_{+}, \epsilon_{-}\right)$of the equations above corresponds to a single preserved supercharge. To determine when such a solution exists, we examine the integrability conditions

$$
\left[D_{\mu}, D_{\nu}\right] \epsilon_{ \pm}=0
$$

Using $\left[D_{\mu}, D_{\nu}\right] \epsilon_{ \pm}=-\frac{1}{4} R_{\mu \nu}^{a b}(J) \gamma_{a b} \epsilon_{ \pm}$, (4.1) and (4.2), the integrability conditions take the form

$$
\begin{array}{r}
A_{\mu \nu} \gamma_{0} \epsilon_{+}=0, \\
B_{\mu \nu} \gamma_{0} \epsilon_{-}+C_{\mu \nu}^{a} \gamma_{a} \epsilon_{+}=0,
\end{array}
$$

where

$$
\begin{aligned}
A_{\mu \nu} & =-\frac{1}{4} R_{\mu \nu}{ }^{a b}(J) \epsilon_{a b}-2 \tau_{[\mu} \partial_{\nu]} S, \\
B_{\mu \nu} & =-\frac{1}{4} R_{\mu \nu}{ }^{a b}(J) \epsilon_{a b}+6 \tau_{[\mu} \partial_{\nu]} S, \\
C_{\mu \nu}^{a} & =-\frac{1}{2} \epsilon_{b}^{a} R_{\mu \nu}{ }^{b}(G)+2 e_{[\mu}^{a} \partial_{\nu]} S-4 S^{2} \epsilon_{b}^{a} e_{[\mu}^{b} \tau_{\nu]} .
\end{aligned}
$$

To arrive at these expressions, we have used the constraints (2.21).

Assuming that $\left(\epsilon_{+}, \epsilon_{-}\right)$span a 4-dimensional spinor space, the necessary and sufficient condition for integrability is $A_{\mu \nu}=B_{\mu \nu}=C_{\mu \nu}^{a}=0$. This is the maximally supersymmetric case with four supercharges, which we analyze further in section 4.1.

To find backgrounds with less than maximal supersymmetry, one may consider imposing further constraints on the Killing spinors, e.g. $\epsilon_{-}=0$. In this case, however, the integrability condition needs to be rederived in the appropriate lower-dimensional subspace of solutions, and may take a different form ${ }^{1}$. We will study examples of such $\frac{1}{2}$-BPS backgrounds with two supercharges in section 4.2.

As we will demonstrate later, the existence of a single supercharge implies the existence of at least one more supercharge, i.e. solutions to the Killing spinor equations always come in pairs. Hence there are no $\frac{1}{4}$-BPS solutions.

\subsection{Maximally supersymmetric solutions}

Backgrounds with completely unbroken supersymmetry admit four real supercharges, or equivalently four linearly independent Killing spinors of the form $\left(\epsilon_{+}, \epsilon_{-}\right)$. To solve the

\footnotetext{
${ }^{1}$ For example, it is easy to convince oneself that setting $\epsilon_{-}=0, A_{\mu \nu}=C_{\mu \nu}^{a}=0$ solves (4.4), but plugging this ansatz back into (4.1) and (4.2) does not guarantee a solution.
} 
integrability condition (4.4), we therefore need to demand $A_{\mu \nu}=B_{\mu \nu}=C_{\mu \nu}^{a}=0$, which implies

$$
\begin{aligned}
\tau_{[\mu} \partial_{\nu]} S & =0, \\
R_{\mu \nu}{ }^{a b}(J) & =0, \\
R_{\mu \nu}{ }^{a}(G) & =8 S^{2} \tau_{[\mu} e_{\nu]}^{a}-4 \epsilon^{a}{ }_{b} e_{[\mu}^{b} \partial_{\nu]} S .
\end{aligned}
$$

Together with the constraint $R_{\mu \nu}(H)=2 \partial_{[\mu} \tau_{\nu]}=0$ (see section 2.2), these equations completely determine the maximally supersymmetric backgrounds. To make contact with the more familiar language of General Relativity, it is useful to translate the constraints (4.9) and (4.10) into conditions on the Riemann tensor constructed from the Christoffel connection,

$$
R_{\nu \rho \sigma}^{\mu}(\Gamma)=-e_{a}^{\mu}\left(\tau_{\nu} R_{\rho \sigma}^{a}(G)+e_{\nu b} R_{\rho \sigma}^{a b}(J)\right) .
$$

Using this expression, we can rewrite the conditions (4.8) through (4.10) as

$$
\begin{aligned}
\tau_{[\mu} \partial_{\nu]} S & =0 \\
R^{\mu}{ }_{\nu \rho \sigma}(\Gamma) & =-8 S^{2} \tau_{\nu} \tau_{[\rho} \delta_{\sigma]}^{\mu}+4 \epsilon^{a}{ }_{b} e_{a}^{\mu} \tau_{\nu} e_{[\rho}^{b} \partial_{\sigma]} S .
\end{aligned}
$$

The Ricci tensor is given by

$$
R_{\mu \nu}=8 S^{2} \tau_{\mu} \tau_{\nu}
$$

This is, in fact, the standard Einstein equation for Newton-Cartan gravity, $R_{\mu \nu}=4 \pi G \rho \tau_{\mu} \tau_{\nu}$ with $S^{2}$ playing the role of the Newtonian mass density $\rho$. Note, however, that here it arises as a condition of maximal supersymmetry, and not through the direct imposition of any equations of motion.

To analyze (4.12) and (4.13) further, we introduce adapted coordinates (see section 2.2). In these coordinates, the first of the two constraints simply becomes

$$
\partial_{i} S=0
$$

To evaluate the second constraint, we first note that (4.14) implies $R_{i j}=0$. The spatial metric $h_{i j}$ is therefore flat, with a possible time dependence:

$$
h_{i j}=g(t) \delta_{i j}
$$

We can use the gauge freedom (2.28) to set

$$
h_{i j}=\delta_{i j}, \quad h^{i j}=\delta^{i j} .
$$

After making this gauge choice, the remaining allowed coordinate transformations are

$$
\begin{aligned}
t & \rightarrow t+\text { const. }, \\
x^{i} & \rightarrow A_{j}^{i}(t) x^{j}+a^{i}(t),
\end{aligned}
$$


where $A_{k}^{i} A_{j}{ }^{k}=\delta_{j}^{i}$. The $A_{j}^{i}$ parametrize time-dependent rotations, while $a^{i}(t)$ corresponds to a Galilean boost. For this reason, the gauge choice $h_{i j}=\delta_{i j}$ is sometimes referred to as choosing "Galilean coordinates" [14]. In these coordinates, the conditions (2.12) determine the spatial metric to take the form

$$
h_{\mu \nu}=\left(\begin{array}{cc}
v^{i} v_{i} & -v^{i} \\
-v^{i} & \delta_{i j}
\end{array}\right) .
$$

Knowing the form of the spatial metric, we can explicitly write down the vielbein and its inverse:

$$
\begin{aligned}
e_{\mu}^{a} & =\left(-v^{a}, \delta_{i}^{a}\right), \\
e_{a}^{\mu} & =\left(0, \delta_{a}^{i}\right)^{T} .
\end{aligned}
$$

We are now ready to write the second integrability condition (4.13) in Galilean coordinates. To express the Riemann tensor explicitly in terms of metric components, first note that the only nonzero connection coefficients are

$$
\begin{aligned}
& \Gamma_{00}^{i}=\partial_{i}\left(m_{0}-\frac{1}{2} h_{00}\right)-\partial_{0}\left(m_{i}-h_{i 0}\right) \equiv \Phi_{i}, \\
& \Gamma_{0 j}^{i}=-\Gamma_{0 i}^{j}=\partial_{[i}\left(m_{j]}-h_{j] 0}\right) \equiv C_{i j}=-C_{j i} .
\end{aligned}
$$

To simplify our discussion, it will be useful to define $C \equiv \frac{1}{2} \epsilon_{i j} C^{i j}$, with $\epsilon_{12}=1$. Using these definitions, the Riemann-constraint (4.13) may be written as

$$
\begin{aligned}
\partial_{i} C & =0 \\
\partial_{j} \Phi_{i}-\epsilon_{i j} \partial_{0} C+\delta_{i j} C^{2} & =4 \delta_{i j} S^{2}+2 \epsilon_{i j} \partial_{0} S .
\end{aligned}
$$

Taking the antisymmetric part of the second equation and using the definitions (4.22) and (4.23), we find $\partial_{0} S=0$, and therefore conclude that $S=$ const.

To summarize, backgrounds admitting four supercharges are given by a degenerate spatial metric $h_{\mu \nu}$ of the form (4.19), and connection coefficients $\Phi_{i}, C$, such that

$$
\begin{aligned}
\partial_{(i} \Phi_{j)}+\delta_{i j}\left(C^{2}-4 S^{2}\right) & =0 \\
\partial_{i} C & =0 \\
S & =\text { const. }
\end{aligned}
$$

Given a specific background, the auxiliary field $S$ required to close the non-relativistic SUSY algebra is found by solving (4.26). Since $S$ is constant, (4.14) demonstrates that maximally supersymmetric solutions are essentially Newtonian cosmologies with a homogeneous matter distribution $\rho=2 S^{2} / \pi G \geq 0$.

\subsubsection{Connection coefficients}

To give a physical interpretation to the connection coefficients $\Phi_{i}$ and $C$, let us consider the geodesic equation in the backgrounds discussed above:

$$
\frac{d^{2} x^{i}}{d t^{2}}+\Phi_{i}+2 C_{i j} \frac{d x^{j}}{d t}=0
$$


We see that $\Phi_{i}$ represents the gravitational force, while $C_{i j}$ is akin to the Coriolis force in a rotating reference frame. Defining a scalar and vector potential via

$$
\begin{aligned}
\varphi & =m_{0}-\frac{1}{2} h_{00}, \\
A_{i} & =m_{i}-h_{i 0},
\end{aligned}
$$

we may use (4.22) and (4.23) to write the two force fields as

$$
\begin{aligned}
\Phi_{i} & =\partial_{i} \varphi-\partial_{0} A_{i}, \\
C_{i j} & =\partial_{[i} A_{j]} .
\end{aligned}
$$

One may then identify $\Phi_{i}$ and $C_{i j}$ in (4.29) as "electric" and "magnetic" - type fields, which are invariant under the gauge transformation $\varphi \rightarrow \varphi+\partial_{0} \lambda, A_{i} \rightarrow A_{i}+\partial_{i} \lambda$.

Finally, note that the vector field $m_{\mu}$ is not part of the metric itself, but only shows up in the expressions (4.22) and (4.23) for the connection coefficients in a given background $h_{\mu \nu}$. Changing $m_{\mu}$ is equivalent to changing the rules for parallel transport in a fixed background.

We can solve the conditions (4.26) through (4.28) explicitly by performing a Galilei transformation (4.18) into a non-rotating coordinate frame, where $C=0$. In this case $A_{i}$ is rotation-free, and we can locally write $A_{i}=\partial_{i} \psi$. The constraint (4.26) then takes the form

$$
\partial_{i} \partial_{j} \hat{\varphi}=4 S^{2} \delta_{i j}
$$

where we introduced a new potential $\hat{\varphi}=\varphi-\partial_{0} \psi$. Taking the trace of this equation, we recover Poisson's equation with a source $\rho=2 S^{2} / \pi G=$ const. However, since (4.32) also contains the additional condition $\left(\partial_{1}^{2}-\partial_{2}^{2}\right) \hat{\varphi}=\partial_{1} \partial_{2} \hat{\varphi}=0$, the solution is further constrained. We find

$$
\hat{\varphi}(x, y)=2 S^{2}\left(x^{2}+y^{2}\right)+c_{1}(t) x+c_{2}(t) y+d(t),
$$

instead of the usual logarithmic solution for a gravitational potential of a homogeneous matter distribution. A particle moving along a geodesic in a maximally supersymmetric background experiences a Newtonian gravitational force

$$
\Phi_{i}=\partial_{i} \hat{\varphi}=4 S^{2} x^{i}+c_{i}(t)
$$

\subsubsection{Killing spinors}

We can explicitly construct all four supercharges of the maximally supersymmetric case by solving (4.1) and (4.2) in the backgrounds constructed above. For backgrounds with $h_{i j}=\delta_{i j}$, the Killing spinor equations take the form

$$
\begin{aligned}
0 & =\partial_{i} \epsilon_{+} \\
0 & =\left[\partial_{0}+\left(\frac{1}{2} C+S\right) \gamma_{0}\right] \epsilon_{+} \\
0 & =\partial_{i} \epsilon_{-}-\left(\frac{1}{2} C+S\right) \gamma_{i} \epsilon_{+}-\frac{1}{2} \epsilon_{a b} \partial_{i} v_{a} \gamma_{b} \epsilon_{+} \\
0 & =\left[\partial_{0}+\left(\frac{1}{2} C-3 S\right) \gamma_{0}\right] \epsilon_{-}+\left(S-\frac{1}{2} C\right) v^{a} \gamma_{a} \epsilon_{+}-\frac{1}{2} \epsilon_{a b}\left(\Phi_{a}+\partial_{0} v_{b}\right) \gamma_{b} \epsilon_{+} .
\end{aligned}
$$


These equations can be solved, provided the integrability conditions found previously hold. The condition $\partial_{\mu} S=\partial_{i} C=0$ guarantees the existence of two linearly independent homogeneous solutions

$$
\epsilon_{+}=0, \quad \epsilon_{-}=e^{-\int d t^{\prime}\left(3 S-\frac{1}{2} C\right) \gamma_{0}} \epsilon_{0},
$$

with $\epsilon_{0}$ an arbitrary constant Majorana spinor. Using (4.26), one can show that there are two additional inhomogeneous solutions:

$$
\epsilon_{+}=e^{-\int d t^{\prime}\left(\frac{1}{2} C+S\right) \gamma_{0}} \epsilon_{0}^{\prime}, \quad \epsilon_{-}=e^{\int d t^{\prime}\left(3 S-\frac{1}{2} C\right) \gamma_{0}} M(t) \epsilon_{0}^{\prime} .
$$

Here $\epsilon_{0}^{\prime}$ is another constant Majorana spinor, and we defined

$$
M(t)=\int^{t} d t^{\prime}\left[e^{t^{t^{\prime}} d t^{\prime \prime}\left(\frac{1}{2} C-3 S\right) \gamma_{0}}\left(\left(\frac{1}{2} C-S\right) v^{a} \gamma_{a}+\frac{1}{2} \epsilon_{a b}\left(\Phi_{a}+\partial_{0} v_{a}\right) \gamma_{b}\right) e^{-\int^{t^{\prime}} d t^{\prime \prime}\left(\frac{1}{2} C+S\right) \gamma_{0}}\right] .
$$

This concludes our discussion of maximally supersymmetric backgrounds.

\section{$4.2 \quad \frac{1}{2}$-BPS solutions}

We now turn to backgrounds that admit only two supercharges. Since we are interested in solving the Killing spinor equations (4.1) and (4.2) in a 2-dimensional subspace $\mathcal{S}$ of the full spinor space spanned by $\left(\epsilon_{+}, \epsilon_{-}\right)$, the integrability condition (4.4) will have to be rederived in the appropriate subspace. For each such space $\mathcal{S}_{i}$, we will be able to give the necessary and sufficient condition for integrability. If we label the set of backgrounds that satisfy this condition by $\mathcal{M}_{i}$, the full set of $\frac{1}{2}$-BPS backgrounds is given by $\bigcup \mathcal{M}_{i}$.

However, since there are of course infinitely many subspaces $\mathcal{S}$, using integrability to find all $\frac{1}{2}$-BPS backgrounds seems impractical. We therefore content ourselves with studying specific examples of $\frac{1}{2}$-BPS solutions by specifying the subspace $\mathcal{S}_{i}$ in which their Killing spinors live, and study integrability for each of them individually.

\subsubsection{Backgrounds with two supercharges of the form $\left(0, \epsilon_{-}\right)$}

We start by considering the case $\epsilon_{+}=0$. The Killing spinor equations (4.1) and (4.2) simplify to a single equation:

$$
\left(D_{\mu}-3 S \tau_{\mu} \gamma_{0}\right) \epsilon_{-}=0 .
$$

This equation is integrable if and only if

$$
R_{\mu \nu}^{a b}(J)=12 \epsilon_{a b} \tau_{[\mu} \partial_{\nu]} S .
$$

In adapted coordinates $R_{i j}{ }^{a b}(J)=0$, which implies $R_{i j}=0$ (see (4.11)). Thus we can again choose Galilean coordinates such that $h_{i j}=\delta_{i j}$. The spatial vielbein and its inverse are given by (4.20) and (4.21), respectively. The nonzero components of the Riemann tensor are

$$
\begin{aligned}
R_{0 j) 0}^{(i}(\Gamma) & =-R_{(j 0}{ }^{i)}(G)+6 \epsilon_{b(i} v^{b} \partial_{j)} S, \\
R_{0 j k}^{i}(\Gamma) & =12 \epsilon_{[k}^{i} \partial_{j]} S, \\
R_{j k 0}^{i}(\Gamma) & =6 \epsilon^{i}{ }_{j} \partial_{k} S .
\end{aligned}
$$


We can once again express the left hand side of these constraints in terms of the connection coefficients $\Phi_{i}=\Gamma_{00}^{i}, C=\frac{1}{2} \epsilon_{i j} \Gamma_{0 j}^{i}$. Since $R_{(j 0}{ }^{i)}(G)$ remains undetermined, the first equation does not impose any further constraints on $\Phi_{i}$ and $C$. Equations (4.45) and (4.46) are equivalent to the condition

$$
\partial_{i} S=\frac{1}{6} \partial_{i} C
$$

To summarize, a given background admits two supercharges of the form $\left(0, \epsilon_{-}\right)$if and only if $R_{i j}=0$. Given a background with arbitrary $\Phi_{i}$ and $C$, one can always choose the auxiliary scalar $S$ such that (4.47) is satisfied.

The Killing spinors in this class of backgrounds can be constructed explicitly by solving (4.42), which now takes the form

$$
\begin{aligned}
& 0=\partial_{i} \epsilon_{-}, \\
& 0=\left[\partial_{0}+\left(\frac{1}{2} C-3 S\right) \gamma_{0}\right] \epsilon_{-} .
\end{aligned}
$$

The second equation can be easily integrated to find the two solutions

$$
\epsilon_{-}=e^{\int d t^{\prime}\left(3 S-\frac{1}{2} C\right) \gamma_{0}} \epsilon_{0}, \quad\left(\epsilon_{+}=0\right),
$$

where $\epsilon_{0}$ is a constant Majorana spinor. The integrability condition (4.47) then guarantees that (4.48) is satisfied as well.

\subsubsection{Backgrounds with two supercharges of the form $\left(\epsilon_{+}, 0\right)$}

Another class of $\frac{1}{2}$-BPS backgrounds is characterized by $\epsilon_{-}=0$. The Killing spinor equations in this case read

$$
\begin{aligned}
\left(D_{\mu}+S \tau_{\mu} \gamma_{0}\right) \epsilon_{+} & =0, \\
\left(\frac{1}{2} \omega_{\mu}^{a} \gamma_{a 0}-S e_{\mu}^{a} \gamma_{a}\right) \epsilon_{+} & =0 .
\end{aligned}
$$

Note that the second equation is purely algebraic. Integrability requires

$$
A_{\mu \nu} \equiv-\frac{1}{4} R_{\mu \nu}^{a b}(J) \epsilon_{a b}-2 \tau_{[\mu} \partial_{\nu]} S=0
$$

as well as

$$
\frac{1}{2} \omega_{\mu}^{a} \gamma_{a 0}-S e_{\mu}^{a} \gamma_{a}=0
$$

The first condition implies $R_{\mu \nu}^{a b}(J)=-4 \epsilon_{a b} \tau_{[\mu} \partial_{\nu]} S$. This condition differs from (4.43) only by a numerical factor, so we again conclude that $R_{i j}=0$, and choose $h_{i j}=\delta_{i j}$. In adapted coordinates, the nonzero components of the Riemann tensor are then

$$
\begin{aligned}
R_{0 j) 0}^{(i} & =-R_{(j 0}{ }^{i)}(G)-2 \epsilon_{b(i} v^{b} \partial_{j)} S \\
R_{0 j k}^{i} & =-4 \epsilon_{[k}^{i} \partial_{j]} S \\
R_{j k 0}^{i} & =-2 \epsilon^{i}{ }_{j} \partial_{k} S .
\end{aligned}
$$


As before, the first equation does not yield any additional constraints. The second and third equation are equivalent to

$$
\partial_{i} S=-\frac{1}{2} \partial_{i} C
$$

which is the analog of (4.47).

We now turn to solving the second integrability condition, (4.54). After evaluating the boost connection $\omega_{\mu}^{a}$ for a metric of the form

$$
h_{\mu \nu}=\left(\begin{array}{cc}
v^{i} v_{i} & -v^{i} \\
-v^{i} & \delta_{i j}
\end{array}\right)
$$

we arrive at the following conditions:

$$
\begin{aligned}
S & =-\frac{1}{2} C+\frac{1}{4} \epsilon^{a b} \partial_{a} v_{b}, \\
\partial_{(a} v_{b)} & =0, \\
\Phi_{a} & =(2 S-C) \epsilon_{a b} v^{b}-\partial_{0} v_{b} .
\end{aligned}
$$

Note that the first two conditions together imply (4.58). The last condition can be rewritten using (4.30) and (4.31) to find

$$
\partial_{a} m_{0}-\partial_{0} m_{a}=-\epsilon_{a b} v^{b} \epsilon^{c d} \partial_{c} m_{d}
$$

Assume that we fix a background metric $h_{\mu \nu}$ by fixing $v^{a}$, such that $\partial_{(a} v_{b)}=0$. Then (4.63) can be viewed as a constraint on the allowed $m_{\mu}$, which determine the choice of connection $\Gamma$ in this background.

To find the two supercharges explicitly, we consider (4.51) and (4.52):

$$
\begin{aligned}
& 0=\partial_{i} \epsilon_{+}, \\
& 0=\left[\partial_{0}+\frac{1}{4} \epsilon^{a b} \partial_{a} v_{b} \gamma_{0}\right] \epsilon_{+} .
\end{aligned}
$$

The solutions are given by

$$
\epsilon_{+}=e^{-\frac{1}{4} \int d t^{\prime} \epsilon^{a b} \partial_{a} v_{b} \gamma_{0}} \epsilon_{0}, \quad\left(\epsilon_{-}=0\right),
$$

with $\epsilon_{0}$ a constant Majorana spinor.

\subsubsection{Backgrounds with two supercharges of the form $\left(\epsilon_{+}, F \epsilon_{+}\right)$}

To complete our discussion of $\frac{1}{2}$-BPS solutions, we consider the case where the 2 dimensional spinor subspace $\mathcal{S}$ is not simply given by $\epsilon_{ \pm}=0$, but is rather spanned by nontrivial linear combinations of $\epsilon_{+}$and $\epsilon_{-}$. We make the ansatz

$$
\epsilon_{-}=F(t, \vec{x}) \epsilon_{+}=F^{\mu}(t, \vec{x}) \gamma_{\mu} \epsilon_{+}
$$


Plugging this ansatz into the Killing spinor equations (4.1) and (4.2), we find

$$
\begin{aligned}
D_{\mu} \epsilon_{+} & =-S \tau_{\mu} \gamma_{0} \epsilon_{+}, \\
b_{\mu} & =-\left(\partial_{\mu}+a_{\mu}\right) F^{a} \gamma_{a}, \\
F^{0} & =0 .
\end{aligned}
$$

where

$$
\begin{aligned}
a_{\mu} & =-\frac{1}{2} \omega_{\mu}^{a b} \gamma_{a b}-2 S \tau_{\mu} \gamma_{0}, \\
b_{\mu} & =\frac{1}{2} \omega_{\mu}^{a} \gamma_{a 0}-S e_{\mu}^{a} \gamma_{a},
\end{aligned}
$$

encode the geometric information about the background.

A nonzero Killing spinor $\epsilon_{+}$exists if and only if (4.68) is integrable, which as we saw previously, requires

$$
R_{\mu \nu}^{a b}(J)=-4 \epsilon_{a b} \tau_{[\mu} \partial_{\nu]} S .
$$

Following the analysis in section 4.2.2, the Riemann components are given by (4.55)-(4.57), and we again find that $h_{i j}=\delta_{i j}$ and

$$
\partial_{i} S=-\frac{1}{2} \partial_{i} C
$$

It is important to recall that the functions $F^{a}$ were introduced to determine a certain subspace $\left(\epsilon_{+}, F \epsilon_{+}\right)$, in which we find the Killing spinors. Therefore, (4.69) should not be seen as a PDE for $F^{a}$; rather, we should think of $F$ as being fixed, and (4.69) as determining the background, encoded in $a_{\mu}$ and $b_{\mu}$. In section 4.2.2, we followed precisely this strategy by choosing $F=0$, which led to $b_{\mu}=0$. For an arbitrary but fixed $F$, there are two Killing spinors of the form

$$
\epsilon_{+}=e^{-\int d t\left(\frac{1}{2} C+S\right) \gamma_{0}} \epsilon_{0}, \quad\left(\epsilon_{-}=F \epsilon_{+}\right),
$$

where $\epsilon_{0}$ is a constant Majorana spinor.

\subsubsection{A nontrivial example}

An example of a nontrivial two-dimensional spinor-subspace $\mathcal{S}$ with $F \neq 0$ is given by

$$
F_{a}=\frac{1}{2} \epsilon_{a b} v_{b}
$$

With this choice, we find the following conditions on the background fields:

$$
\begin{aligned}
S & =-\frac{1}{2} C, \\
\Phi_{a} & =0 .
\end{aligned}
$$

Following (4.75), the two supercharges take the form

$$
\left(\epsilon_{+}, \epsilon_{-}\right)=\left(\epsilon_{0}, \frac{1}{2} \epsilon_{a b} v_{b} \gamma^{a} \epsilon_{0}\right)
$$

Notice that if the background satisfies $\partial_{\mu} C=0$, all the conditions for maximal supersymmetry (4.27)-(4.28) are satisfied as well, and supersymmetry is enhanced from two to four supercharges. 


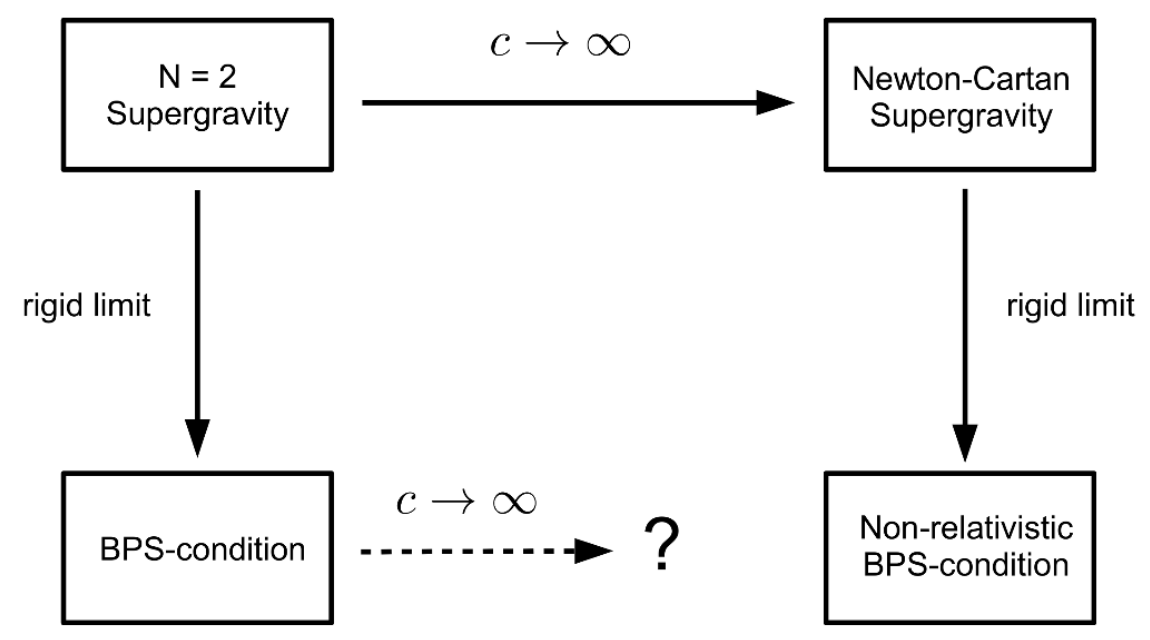

Figure 2. The non-relativistic BPS-condition is obtained by taking the rigid limit of NewtonCartan supergravity, which arises as a non-relativistic limit of $\mathcal{N}=2$ supergravity. One may ask whether the same result can be obtained by reversing the order of the non-relativistic and rigid limits.

\section{Rigid backgrounds of $\mathcal{N}=2$ supergravity}

In the previous section we have found rigid backgrounds of non-relativistic supergravity. It is interesting to ask whether the same result can be obtained by taking the non-relativistic limit of rigid backgrounds of $\mathcal{N}=2$ supergravity [17, 28, 36-38] (see figure 2).

With this question in mind, in this section we revisit the computation of rigid backgrounds of relativistic supergravity, with the purpose of taking the non-relativistic limit presented in [1] later on. We start by recalling the variation of the gravitino under supersymmetry transformations parametrized by the Majorana spinors $\eta_{i}$, and an R-transformation parametrized by $\rho$ (see (3.1)):

$$
\delta \Psi_{\mu}^{i}=\mathcal{D}_{\mu}^{i j} \eta_{j}-\frac{1}{2} \epsilon^{i j} \bar{\Psi}_{[\mu i} \Psi_{\nu] j}+\Psi_{\mu j} \rho,
$$

where the operator $\mathcal{D}_{\mu}^{i j}$ given by

$$
\mathcal{D}_{\mu}^{i j} \equiv \nabla_{\mu} \delta^{i j}-\gamma_{\mu} D \delta^{i j}+V_{\mu} \epsilon^{i j}+\frac{1}{4} \gamma_{\mu} \gamma^{\rho \sigma} F_{\rho \sigma} \epsilon^{i j} .
$$

$F_{\rho \sigma}$ is the field strength of the gauge field $M_{\sigma}, F=d M$. In the rigid limit we set $\Psi_{\mu}^{i}=$ $\delta \Psi_{\mu}^{i}=0$, which implies

$$
\mathcal{D}_{\mu}^{i j} \eta_{j}=0 .
$$

Rigid supersymmetric backgrounds are given by a choice of $g_{\mu \nu}$, as well as auxiliary fields $V_{\mu}, M_{\mu}, D$ such that the Killing spinor equation (5.3) is integrable. We determine when solutions to (5.3) exist by studying its integrability condition, which takes the general form

$$
0=\left[\mathcal{D}_{\mu}^{i j}, \mathcal{D}_{\nu}^{j k}\right] \eta_{k}=\left(A_{\mu \nu} \delta^{i k}+B_{\mu \nu}{ }^{\lambda} \gamma_{\lambda} \delta^{i k}+C_{\mu \nu} \epsilon^{i k}+D_{\mu \nu}{ }^{\lambda} \gamma_{\lambda} \epsilon^{i k}\right) \eta_{k}
$$


In our case, we find

$$
\begin{aligned}
A_{\mu \nu} & =0 \\
B_{\mu \nu}{ }^{\lambda} & =-\frac{1}{4} R_{\mu \nu \rho \sigma} \epsilon^{\rho \sigma \lambda}+2 \delta_{[\mu}^{\lambda} \partial_{\nu]} D-2 \epsilon_{\mu \nu}{ }^{\lambda} D^{2}+\frac{1}{2} \epsilon^{\sigma \tau \lambda} F_{\mu \sigma} F_{\nu \tau}, \\
C_{\mu \nu} & =2 \partial_{[\mu} V_{\nu]}+\frac{1}{2} \epsilon^{\rho \sigma}{ }_{[\mu} \nabla_{\nu]} F_{\rho \sigma}, \\
D_{\mu \nu}{ }^{\lambda} & =\delta_{[\mu}^{\lambda} \epsilon_{\nu]}^{\rho \sigma} D F_{\rho \sigma}-\frac{1}{2} \nabla^{\lambda} F_{\mu \nu} .
\end{aligned}
$$

We focus on maximally supersymmetric backgrounds, which admit four real supercharges. In this context, just as in the non-relativistic case, there are four linearly independent Killing spinors, so all terms in (5.4) should vanish independently. $A_{\mu \nu}=0$ is already guaranteed. $B_{\mu \nu}{ }^{\lambda}=0$ imposes

$$
\begin{aligned}
R_{\mu \nu} & =-8 D^{2} g_{\mu \nu}+g^{\alpha \beta} F_{\alpha \mu} F_{\beta \nu}, \\
D & =\text { const. }
\end{aligned}
$$

These constraints are found by solving for the Riemann tensor and contracting it with the metric, and by contracting Riemann with the Levi-Civita tensor. It is convenient to express the constraints in terms of the dual field strength, defined by

$$
\begin{aligned}
F_{\mu \nu} & =\epsilon_{\mu \nu \rho} f^{\rho}, \\
f^{\rho} & =-\frac{1}{2} F_{\mu \nu} \epsilon^{\mu \nu \rho} .
\end{aligned}
$$

With this redefinition, the Ricci tensor (5.9) is then given by

$$
R_{\mu \nu}=-8 D^{2} g_{\mu \nu}+f_{\mu} f_{\nu}-f^{\alpha} f_{\alpha} g_{\mu \nu} .
$$

The condition $C_{\mu \nu}=0$ imposes a relation between the field strength of $V_{\mu}$ and $F_{\mu \nu}$,

$$
2 \partial_{[\mu} V_{\nu]}=-\frac{1}{2} \epsilon_{[\mu}^{\rho \sigma} \nabla_{\nu]} F_{\rho \sigma}
$$

Using (5.11), we rewrite (5.13) as an expression relating the field strength of $f_{\mu}$ and that of $V_{\mu}$,

$$
2 \partial_{[\mu} V_{\nu]}=-\partial_{[\mu} f_{\nu]} .
$$

Thus, $f_{\mu}$ and $V_{\mu}$ are proportional up to the addition of an arbitrary closed 1-form, which would not change (5.14),

$$
f_{\mu}=-2 V_{\mu}+\lambda_{\mu}^{\prime} .
$$

Finally, the condition $D_{\mu \nu}{ }^{\lambda}=0$ implies

$$
\nabla_{\mu} f_{\nu}+2 D \epsilon_{\mu \nu \rho} f^{\rho}=0 .
$$

The symmetric and antisymmetric parts of (5.16) are

$$
\begin{aligned}
& \nabla_{(\mu} f_{\nu)}=0, \\
& \nabla_{[\mu} f_{\nu]}=-2 D \epsilon_{\mu \nu \rho} f^{\rho}=-2 D F_{\mu \nu} .
\end{aligned}
$$


Equation (5.17) shows that $f^{\mu}$ is a Killing vector, while (5.18) implies that the field strength of $f_{\mu}$ is proportional to $F_{\mu \nu}$. Equivalently, $f_{\mu}$ and $M_{\mu}$ are related by

$$
f_{\mu}=-4 D M_{\mu}+\lambda_{\mu}
$$

where $\lambda_{\mu}$ is an arbitrary closed 1-form. Again, this ambiguity shows up since the addition of a closed 1-form to $f_{\mu}$ does not change the constraint (5.18). Another consequence of $(5.16)$ is

$$
\nabla_{\mu} f^{\nu} f_{\nu}=-4 D \epsilon_{\mu \nu \rho} f^{\nu} f^{\rho}=0 .
$$

That is, in addition to being a Killing vector, $f^{\mu}$ has constant norm. Hence, the possible backgrounds are given by $f_{\mu}=0$ as well as $f_{\mu} \neq 0$ with $f_{\mu}$ timelike, spacelike, or null.

\subsection{The $f_{\mu}=0$ case}

For a vanishing $f_{\mu}$, the Ricci tensor reduces to

$$
R_{\mu \nu}=-8 D^{2} g_{\mu \nu}
$$

The background is locally $\mathrm{AdS}_{3}$, with radius $\ell_{A}=\frac{1}{2|D|}$. Equations (5.15) and (5.19) imply that $M_{\mu}$ and $V_{\mu}$ are closed and undetermined, i.e., they are pure gauge.

\subsection{The timelike case}

For $f^{\mu}$ a constant norm timelike Killing vector, we introduce adapted coordinates such that

$$
f^{\mu} \partial_{\mu}=\frac{\partial}{\partial t}
$$

Normalizing $f^{\mu}$ by taking $f^{2}=-N^{2}<0$, where $N$ is a non-negative constant, we can write the most general metric admitting a timelike Killing direction as

$$
d s^{2}=-N^{2}(d t+u)^{2}+d s_{(2)}^{2},
$$

where $u=u_{i}(x, y) d x^{i}$ and

$$
d s_{(2)}^{2}=e^{2 \sigma}\left(d x^{2}+d y^{2}\right)
$$

is a conformally flat 2-dimensional metric with $\sigma=\sigma(x, y)$. The metric (5.23) describes a fibration of a timelike coordinate over the 2-dimensional metric $d s_{(2)}^{2}$. In the adapted coordinate system, $f_{\mu}$ is given by

$$
f_{\mu}=\left(-N^{2},-N^{2} u_{i}\right)
$$

The integrability constraints (5.12) and (5.16) impose

$$
\begin{aligned}
e^{-2 \sigma}\left(\partial_{1}^{2}+\partial_{2}^{2}\right) \sigma & =\left(16 D^{2}-N^{2}\right) . \\
\frac{4 D}{N} & =\left(\partial_{1} u_{2}-\partial_{2} u_{1}\right) e^{-2 \sigma},
\end{aligned}
$$

The first constraint is Liouville's equation, the left hand side of which describes $R_{(2)}$, the Ricci scalar of the 2-dimensional base metric $d s_{(2)}^{2}$. Liouville's equation has well known 
solutions; once solved one can insert the solution $\sigma(x, y)$ into the second constraint and solve for $u$, which specifies the way $\mathbb{R}$ is fibered over the spatial manifold.

We focus on the Ricci scalar,

$$
R_{(2)}=-2 e^{-2 \sigma}\left(\partial_{1}^{2}+\partial_{2}^{2}\right) \sigma=2\left(N^{2}-16 D^{2}\right) .
$$

In the last step we used (5.26). We see that the curvature of the 2-dimensional manifold is constant. Note that $N$ contributes positively to the curvature while $D$ contributes negatively. Thus, the supersymmetric backgrounds with a timelike $f_{\mu}$ are fibrations of a timelike direction over a 2-dimensional manifold that is locally $S^{2}, \mathbb{H}^{2}$, or $\mathbb{R}^{2}$ :

$$
\begin{aligned}
& \mathbb{R} \widetilde{\times} S^{2}, \text { if } N>4|D|, \\
& \mathbb{R} \widetilde{\times} \mathbb{R}^{2}, \text { if } N=4|D|, \\
& \mathbb{R} \widetilde{\times} \mathbb{H}^{2}, \text { if } N<4|D| .
\end{aligned}
$$

Up to coordinate transformations, the metric can be obtained by solving (5.26) explicitly:

$$
d s^{2}=-N^{2}\left(d t+\frac{4 D}{N} \frac{2 r^{2}}{1+\left(N^{2}-16 D^{2}\right) r^{2}} d \phi\right)^{2}+\left(\frac{2}{1+\left(N^{2}-16 D^{2}\right) r^{2}}\right)^{2}\left(d r^{2}+r^{2} d \phi^{2}\right) .
$$

For $N>4|D|$, we can transform to spherical coordinates, so that

$$
\begin{aligned}
d s^{2} & =L^{2}\left[-(d \tau-4 D L \cos \chi d \phi)^{2}+d \chi^{2}+\sin ^{2} \chi d \phi^{2}\right], \\
f & =-N L(d \tau-4 D L \cos \chi d \phi),
\end{aligned}
$$

where $L^{2}=\left(N^{2}-16 D^{2}\right)^{-1}$. When $D=0$, this metric reduces to that of a product space $\mathbb{R} \times S^{2}$. For $N=4|D|$, the space is flat, and we have

$$
\begin{aligned}
d s^{2} & =N^{-2}\left[-\left(d \tau+\frac{1}{2} \chi^{2} d \phi\right)^{2}+d \chi^{2}+\chi^{2} d \phi^{2}\right] . \\
f & =-\left(d \tau+\frac{1}{2} \chi^{2} d \phi\right)
\end{aligned}
$$

Finally, for $N<4|D|$, we obtain instead

$$
\begin{aligned}
d s^{2} & =L^{2}\left[-(d \tau+4 D L \cosh \chi d \phi)^{2}+d \chi^{2}+\sinh ^{2} \chi d \phi^{2}\right], \\
f & =-N L(d \tau+4 D L \cosh \chi d \phi),
\end{aligned}
$$

where $L^{2}=\left(16 D^{2}-N^{2}\right)^{-1}$. Note that here we cannot obtain a direct product space by setting $D=0$ because of the strict inequality $|D|>N / 4 \geq 0$.

\subsection{The spacelike case}

For $f^{2}>0, f^{\mu}$ is a spacelike Killing vector; again we can introduce adapted coordinates such that

$$
f^{\mu} \partial_{\mu}=\frac{\partial}{\partial y}
$$


The most general metric admitting a spacelike Killing vector is

$$
\begin{aligned}
d s^{2} & =e^{2 \sigma}\left(-d t^{2}+d x^{2}\right)+f^{2}(d y+u)^{2} \\
\sigma & =\sigma(t, x), u=u_{\alpha}(t, x) d x^{\alpha}, \alpha=0,1 .
\end{aligned}
$$

In the coordinates (5.37) $f_{\mu}$ is

$$
f_{\mu}=\left(f^{2} u_{0}, f^{2} u_{1}, f^{2}\right) .
$$

The metric (5.37) describes a fibration of a spacelike coordinate over a conformally flat Lorentzian manifold. Note that the spacelike and timelike cases can be related via analytic continuation.

The integrability conditions (5.12) and (5.16) impose the constraints

$$
\begin{aligned}
e^{-2 \sigma}\left(\partial_{0}^{2}-\partial_{1}^{2}\right) \sigma & =-\left(16 D^{2}+f^{2}\right) . \\
-\frac{4 D}{f} & =\left(\partial_{0} u_{1}-\partial_{1} u_{0}\right) e^{-2 \sigma} .
\end{aligned}
$$

In complete analogy with the timelike case, the first equation determines the curvature of the 2-dimensional metric $d s_{(2)}^{2}$ while the second one describes the fibration. The Ricci scalar $R_{(2)}$ is given by

$$
R_{(2)}=2 e^{-2 \sigma}\left(\partial_{0}^{2}-\partial_{1}^{2}\right) \sigma=-2\left(16 D^{2}+f^{2}\right) .
$$

In the last step we used (5.39). Unlike the timelike case, we see that both $D^{2}$ and $f^{2}$ contribute negatively to the curvature. The solution is again a fibration of the real line over a 2-dimensional manifold, but now the only possible $2 \mathrm{D}$ manifold is $\mathrm{AdS}_{2}$. Thus, the supersymmetric background allowing for a spacelike Killing vector is $\mathbb{R} \widetilde{\times} \operatorname{AdS}_{2}$.

\subsection{The null case}

Finally, we consider the case where $f^{\mu}$ is a null Killing vector. We define adapted coordinates $(u, v, x)$ such that

$$
f^{\mu} \partial_{\mu}=\frac{\partial}{\partial v}
$$

Any metric with a null Killing direction $v$ can be written as

$$
d s^{2}=H^{-1}\left(\mathcal{F} d u^{2}+2 d u d v\right)+e^{2 \sigma} d x^{2},
$$

where $H, \mathcal{F}$ and $\sigma$ are functions of $u$ and $x$ only. The integrability constraints (5.12) and (5.16) translate into the following differential equations for the metric functions:

$$
\begin{gathered}
\partial_{x} \log H=4 D e^{\sigma}, \\
\partial_{x}^{2} \mathcal{F}-\partial_{x} \mathcal{F}\left(\partial_{x} \log H+\partial_{x} \sigma\right)+2 H e^{2 \sigma}\left[\partial_{u}^{2} \sigma+\left(\partial_{u} \sigma\right)^{2}+\partial_{u} \sigma \partial_{u} \log H+H^{-2}\right]=0 .
\end{gathered}
$$

This system can be solved by first using (5.44) to express $\sigma$ in terms of $H$, plugging the result into (5.45), and then integrating the resulting equation to find $\mathcal{F}(u, x)$. However, the 
solution is cumbersome and not particularly illuminating. We therefore content ourselves with giving a nontrivial example: consider the case $H(u, x)=1$, which implies $D=0$. In this case, $\sigma$ is arbitrary, and we may choose $\sigma=0$. The solution to (5.45) is then given by

$$
\mathcal{F}(u, x)=-x^{2}-a(u) x-b(u),
$$

with $a(u), b(u)$ being integration constants. The metric reads

$$
d s^{2}=2 d u d v-\left[x^{2}+a(u) x+b(u)\right] d u^{2}+d x^{2} .
$$

This is a plane-fronted wave in Brinkmann coordinates.

\section{Non-relativistic limit of $\mathcal{N}=2$ supergravity}

Given the supersymmetric backgrounds of $\mathcal{N}=2$ supergravity, it is instructive to study how they connect to the non-relativistic supersymmetric solutions of section 4 in the nonrelativistic limit proposed in [1]. Recall the expansions (3.3) of the background fields:

$$
\begin{aligned}
E_{\mu}^{A} & =\delta_{0}^{A}\left(\omega \tau_{\mu}+\frac{1}{2 \omega} m_{\mu}\right)+\delta_{a}^{A} e_{\mu}^{a}, \\
M_{\mu} & =\omega \tau_{\mu}-\frac{1}{2 \omega} m_{\mu}, \\
D & =\frac{S}{\omega} .
\end{aligned}
$$

In addition, we will also need the inverse vielbein, which we obtain perturbatively in $1 / \omega$ :

$$
E_{A}^{\mu}=\delta_{A}^{a}\left(e_{a}^{\mu}-\frac{1}{2 \omega^{2}} m_{\nu} e_{a}^{\nu} \tau^{\mu}+\mathcal{O}\left(\omega^{-4}\right)\right)+\frac{1}{\omega} \delta_{A}^{0}\left(\tau^{\mu}-\frac{1}{2 \omega^{2}} m_{\nu} \tau^{\nu} \tau^{\mu}+\mathcal{O}\left(\omega^{-4}\right)\right)
$$

All other bosonic fields can be expanded in inverse powers of $\omega$. For example,

$$
\begin{aligned}
V_{\mu} & =V_{\mu}^{(0)}+\frac{1}{\omega} V_{\mu}^{(-1)}+\cdots, \\
f_{\mu} & =f_{\mu}^{(0)}+\frac{1}{\omega} f_{\mu}^{(-1)}+\cdots .
\end{aligned}
$$

In the derivation of the Newton-Cartan supergravity theory [1], it was necessary to impose the constraints (3.8) through (3.10), to eliminate divergences. To see if the non-relativistic backgrounds of section 4 could possibly arise as the non-relativistic $(\omega \rightarrow \infty)$ limit of relativistic solutions, we first check if the integrability conditions (5.15) and (5.19) are consistent with (3.9). Taking $\omega \rightarrow \infty$, we find:

$$
V_{\mu}=2 D M_{\mu}-\frac{1}{2}\left(\lambda_{\mu}-\lambda_{\mu}^{\prime}\right) \rightarrow 2 S \tau_{\mu}-\frac{1}{2}\left(\lambda_{\mu}^{(0)}-\lambda_{\mu}^{\prime(0)}\right) .
$$

We see that relativistic integrability implies a relation between the auxiliary fields in the non-relativistic limit. However, there is an ambiguity, parametrized by the closed form $\lambda_{\mu}-\lambda_{\mu}^{\prime}$. The consistency condition (3.9) corresponds to the specific gauge choice $\lambda_{\mu}^{(0)}-$ $\lambda_{\mu}^{\prime(0)}=8 S \tau_{\mu}$. 
The integrability condition (5.12), which fixes the Ricci tensor, can be evaluated in the $\omega \rightarrow \infty$ limit as well:

$$
R_{\mu \nu} \rightarrow 8 S^{2} \tau_{\mu} \tau_{\nu}+\lambda_{\alpha}^{(-1)} \lambda_{\beta}^{(-1)} \eta^{a b} e_{a}^{\alpha} e_{b}^{\beta} \tau_{\mu} \tau_{\nu}
$$

This expression differs from the non-relativistic integrability condition (4.14) only by a $\lambda_{\mu^{-}}$ dependent contribution. The extra contribution can once again be interpreted as a gauge choice in the definition of the fields, $f_{\mu}, M_{\mu}, V_{\mu}$ : the particular choice $\lambda_{\mu}^{(-1)}=0$ yields equation (4.14).

\subsection{Killing spinor equation}

The difference between the non-relativistic limit of the $\mathcal{N}=2$ backgrounds and the nonrelativistic solutions found directly within Newton-Cartan supergravity can be analyzed more systematically by applying the $\omega \rightarrow \infty$ limit (see section 3 ) directly to the relativistic Killing spinor equations (5.3), which we recall here for convenience:

$$
\nabla_{\mu} \eta^{i}+V_{\mu} \epsilon^{i j} \eta_{j}-\gamma_{\mu} D \eta^{i}+\frac{1}{4} \gamma_{\mu} \gamma^{\rho \sigma} F_{\rho \sigma} \epsilon^{i j} \eta_{j}=0
$$

Note that the covariant derivative is given by $\nabla_{\mu}=\partial_{\mu}-\frac{1}{4} \Omega_{\mu}^{A B} \gamma_{A B}$. We first rewrite (6.6) in terms of the spinors $\eta_{ \pm}(3.4)$ :

$$
\begin{aligned}
\left(\partial_{\mu}-\frac{1}{4} \Omega_{\mu}^{a b} \gamma_{a b}\right) & \eta_{+}-\frac{1}{2} \Omega_{\mu}^{0 a} \gamma_{0 a} \eta_{-}-D \gamma_{\mu} \eta_{-}-D E_{\mu 0} \gamma_{0}\left(\eta_{-}-\eta_{+}\right) \\
& -\left(V_{\mu}+\frac{1}{2} f_{\mu}\right) \gamma_{0} \eta_{+}+\frac{1}{2} F_{\mu \nu} \gamma^{\nu} \gamma_{0} \eta_{-}-\frac{1}{2} F_{\mu \nu} E_{0}^{\nu}\left(\eta_{+}+\eta_{-}\right)=0 \\
\left(\partial_{\mu}-\frac{1}{4} \Omega_{\mu}^{a b} \gamma_{a b}\right) & \eta_{-}-\frac{1}{2} \Omega_{\mu}^{0 a} \gamma_{0 a} \eta_{+}-D \gamma_{\mu} \eta_{+}+D E_{\mu 0} \gamma_{0}\left(\eta_{-}-\eta_{+}\right) \\
& +\left(V_{\mu}+\frac{1}{2} f_{\mu}\right) \gamma_{0} \eta_{-}-\frac{1}{2} F_{\mu \nu} \gamma^{\nu} \gamma_{0} \eta_{+}+\frac{1}{2} F_{\mu \nu} E_{0}^{\nu}\left(\eta_{+}+\eta_{-}\right)=0
\end{aligned}
$$

We can expand these equations in powers of $\omega$ by using the redefinitions (3.6) and (3.7) for the spin/boost-connection and (3.5) for the Killing spinors, and also expanding the auxiliary fields according to (6.3). The resulting equations are

$$
\begin{aligned}
& 0= \sqrt{\omega}\left[\left(\partial_{\mu}-\frac{1}{4} \omega_{\mu}^{a b} \gamma_{a b}\right) \epsilon_{+}-S \tau_{\mu} \gamma_{0} \epsilon_{+}-\left(V_{\mu}^{(0)}+\frac{1}{2} f_{\mu}^{(0)}\right) \gamma_{0} \epsilon_{+}\right. \\
&\left.\quad+\frac{1}{2} \tau_{\mu} e_{a}^{\nu} f_{\nu}^{(0)} \epsilon_{-}-\frac{1}{2} \epsilon_{a b} e_{\mu}^{a} e_{b}^{\nu} f_{\nu}^{(0)} \epsilon_{+}\right] \\
&+ \frac{1}{\sqrt{\omega}}\left[-\left(V_{\mu}^{(-1)}+\frac{1}{2} f_{\mu}^{(-1)}\right) \gamma_{0} \epsilon_{+}+\tau_{\mu} e_{a}^{\nu} f_{\nu}^{(-1)} \gamma^{a} \epsilon_{-}-\frac{1}{2} \epsilon_{a b} e_{\mu}^{a} e_{b}^{\nu} f_{\nu}^{(-1)} \epsilon_{+}\right]+\mathcal{O}\left(\omega^{-\frac{3}{2}}\right) \\
& 0= \frac{\omega^{\frac{3}{2}}}{2} \tau_{\mu} e_{a}^{\nu} f_{\nu}^{(0)} \gamma^{a} \epsilon_{+}-\frac{\sqrt{\omega}}{2} \tau_{\mu} e_{a}^{\nu} f_{\nu}^{(-1)} \gamma^{a} \epsilon_{+} \\
&+ \frac{1}{\sqrt{\omega}}\left[\left(\partial_{\mu}-\frac{1}{4} \omega_{\mu}^{a b} \gamma_{a b}\right) \epsilon_{-}-S \tau_{\mu} \gamma_{0} \epsilon_{-}+\left(V_{\mu}^{(0)}+\frac{1}{2} f_{\mu}^{(0)}\right) \gamma_{0} \epsilon_{-}+\frac{1}{2} \omega_{\mu}^{a} \gamma_{a 0} \epsilon_{+}\right. \\
&\left.\quad-S e_{\mu}^{a} \gamma_{a} \epsilon_{+}-\frac{1}{2} F_{\mu \nu}^{(-1)} e_{a}^{\nu} \gamma^{a} \gamma_{0} \epsilon_{+}\right]+\mathcal{O}\left(\omega^{-\frac{3}{2}}\right) .
\end{aligned}
$$


Here we have used the definition $F_{\mu \nu}=\epsilon_{\mu \nu \rho} f^{\rho}=E_{\mu}^{A} E_{\nu}^{B} E^{\rho C} \epsilon_{A B C} f_{\rho}$ to expand $F_{\mu \nu}$ in powers of $\omega$ as well. We see that the Killing spinor equation has split up into terms that are singular/non-singular in the non-relativistic limit $\omega \rightarrow \infty$. In the full supergravity approach, the $\mathcal{O}(\sqrt{\omega})$ - and $\mathcal{O}\left(\frac{1}{\sqrt{\omega}}\right)$-terms would correspond to the variations of $\psi_{\mu+}$ and $\psi_{\mu-}$, respectively. Here we have already set $\delta \psi_{\mu \pm}=0$ in the beginning, so (6.9) and (6.10) lead to Killing spinor equations, plus constraints. Solving (6.9) and (6.10) order by order in large $\omega$, and neglecting $\mathcal{O}\left(\omega^{-\frac{3}{2}}\right)$ terms, we find five independent equations: there are three constraint equations,

$$
\begin{aligned}
\left(V_{\mu}^{(-1)}+\frac{1}{2} f_{\mu}^{(-1)}\right) \gamma_{0} \epsilon_{+}-\tau_{\mu} e_{a}^{\nu} f_{\nu}^{(-1)} \gamma^{a} \epsilon_{-}+\frac{1}{2} \epsilon_{a b} e_{\mu}^{a} e_{b}^{\nu} f_{\nu}^{(-1)} \epsilon_{+} & =0 \\
\tau_{\mu} e_{a}^{\nu} f_{\nu}^{(0)} \gamma^{a} \epsilon_{+} & =0 \\
\tau_{\mu} e_{a}^{\nu} f_{\nu}^{(-1)} \gamma^{a} \epsilon_{+} & =0 .
\end{aligned}
$$

Making no further assumptions about the form or number of supercharges, these conditions need to hold for all $\epsilon_{+}$and $\epsilon_{-}$. We thus conclude that

$$
V_{\mu}^{(-1)}+\frac{1}{2} f_{\mu}^{(-1)}=0, \quad e_{a}^{\nu} f_{\nu}^{(0)}=e_{a}^{\nu} f_{\nu}^{(-1)}=0 .
$$

Using these constraints, we obtain the remaining two equations from (6.9) and (6.10):

$$
\begin{aligned}
D_{\mu} \epsilon_{+} & =S \tau_{\mu} \gamma_{0} \epsilon_{+}+\frac{1}{2} \lambda_{\mu}^{\prime(0)} \gamma_{0} \epsilon_{+} \\
D_{\mu} \epsilon_{-} & =S \tau_{\mu} \gamma_{0} \epsilon_{-}-\frac{1}{2} \lambda_{\mu}^{\prime(0)} \gamma_{0} \epsilon_{-}-\frac{1}{2} \omega_{\mu}^{a} \gamma_{a 0} \epsilon_{+} \\
& +S e_{\mu}^{a} \gamma_{a} \epsilon_{+}+\frac{1}{2} \tau_{\mu}\left(e_{a}^{\nu} f_{\nu}^{(-2)}-\frac{1}{2} m_{\sigma} e_{a}^{\sigma} \tau^{\nu} f_{\nu}^{(0)}\right) \epsilon_{+} .
\end{aligned}
$$

Here $D_{\mu}=\partial_{\mu}-\frac{1}{4} \omega_{\mu}^{a b} \gamma_{a b}$, and $\lambda_{\mu}^{\prime}=2 V_{\mu}+f_{\mu}$ is the undetermined closed form introduced in (5.15). To obtain the last term in the second equation, we have further expanded $F_{\mu \nu}$ in powers of $\omega$ using $F_{\mu \nu}=\epsilon_{\mu \nu \rho} f^{\rho}$ as before.

Comparing the differential equations (6.15) and (6.16) with the non-relativistic Killing spinor equations (4.1) and (4.2), we see that in general they do not agree. Backgrounds that allow spinor solutions of (6.15) and (6.16) are in general not identical to the rigid supersymmetric backgrounds we studied in section 4. However, if we choose

$$
\begin{aligned}
e_{a}^{\nu} f_{\nu}^{(-2)}-\frac{1}{2} m_{\sigma} e_{a}^{\sigma} \tau^{\nu} f_{\nu}^{(0)} & =0 \\
\lambda_{\mu}^{\prime(0)} & =-4 S \tau_{\mu},
\end{aligned}
$$

we reproduce the non-relativistic Killing spinor equations studied previously. This means that the backgrounds allowing for solutions of (6.15) and (6.16) are a superset of the maximally supersymmetric solutions of Newton-Cartan supergravity (see figure 1 in the introduction).

The difference between the two sets of spinor equations is due the different order of limits used in their derivation. Recall that to derive (6.15) and (6.16), we first took the 
rigid limit $\Psi_{\mu}, \delta \Psi_{\mu} \rightarrow 0$, and then the non-relativistic limit $\omega \rightarrow \infty$. On the other hand, the Newton-Cartan supergravity theory of [1] was derived by taking $\omega \rightarrow \infty$ first. In this limit, there are singular terms that arise in the supergravity transformations with nonzero gravitini. To obtain a consistent theory, these singular terms have to be eliminated by imposing the following conditions on the auxiliary fields (see (3.9) and (3.10)) [1]:

$$
\hat{F}_{\mu \nu}=0, \quad V_{\mu}=-2 \tau_{\mu} S .
$$

In the rigid limit, the first condition becomes $f_{\mu}=0$. With these constraints, equations (6.17) and (6.18) are satisfied identically, and we obtain the non-relativistic Killing spinor equations (4.1) and (4.2). Since (6.15) and (6.16) were not derived as a rigid limit of a consistent non-relativistic supergravity theory, we expect the constraints (6.19) to reemerge as a consistency condition if one attempts to couple the rigid supersymmetric theory to gravity.

Note, in particular, that the constraint $f_{\mu}=0$ is very strong, as it ought to be imposed before taking the non-relativistic limit if we wish to remain within the Newton-Cartan supergravity theory of [1]. For maximally supersymmetric backgrounds, this restricts the relativistic starting point to be the $f_{\mu}=0$ case of section 5.1. Defining $g_{\mu \nu}=-\omega^{2} \tau_{\mu} \tau_{\nu}+h_{\mu \nu}$ and substituting into the Einstein condition (5.21) then gives

$$
R_{\mu \nu}=8 S^{2} \tau_{\mu} \tau_{\nu}-8 D^{2} h_{\mu \nu}
$$

where we have taken $S=\omega D$. Taking $\omega \rightarrow \infty$ along with $D \rightarrow 0$ while holding $S$ fixed then reproduces the Ricci condition (4.14) for maximally supersymmetric solutions of the non-relativistic theory.

\section{Discussion}

In contrast to the maximally supersymmetric case, where we were able to construct all non-relativistic backgrounds explicitly, our discussion of $\frac{1}{2}$-BPS solutions in section 4.2 was limited to providing examples of such backgrounds. To find all backgrounds with reduced supersymmetry, it would be interesting to carry out an analysis using spinor bilinears, to find the necessary and sufficient conditions for preserving a single supercharge (see appendix B for such an analysis in the relativistic case). Nevertheless, the three general cases studied in sections 4.2.1, 4.2.2, and 4.2.3 essentially capture all possible $\frac{1}{2}$-BPS solutions. We saw that in each of these three cases, integrability demands that $R_{i j}=0$, so we can conclude that a necessary condition for non-relativistic supersymmetry is that spatial slices are flat. ${ }^{2}$ It would be interesting to see if this continues to be true in higher dimensions, or if it is possible to allow for nonzero curvature of spatial slices.

In our analysis of non-relativistic $\frac{1}{2}$-BPS solutions, which admit two supercharges, we may equally have started by assuming only the form of a single supercharge (e.g. $\left(\epsilon_{+}, 0\right)$ ). After solving the integrability conditions in the appropriate subspace of the 4-dimensional

\footnotetext{
${ }^{2}$ The ansatz in section 4.2 .3 can be slightly generalized to $\epsilon_{-}=\left(F^{\mu}(t, \vec{x}) \gamma_{\mu}+G(t, \vec{x})\right) \epsilon_{+}$. However, the integrability condition of (4.68) and thus the conclusion $R_{i j}=0$ still remain the same.
} 
space of spinors, we saw that Killing spinors necessarily come in pairs, and are characterized by a two component Majorana spinor $\epsilon_{0}$. Hence a single supercharge is automatically enhanced to two supercharges, and there are no $\frac{1}{4}$-BPS solutions. This is a familiar feature from relativistic supersymmetry [30,39] (see also appendix B).

In order to make contact with the backgrounds studied in the context of non-relativistic holography, such as Lifshitz and Schrödinger spacetimes, it is necessary to extend the analysis presented here by including nonzero torsion into the supergravity theory. A torsionful version of Newton-Cartan supergravity has recently been constructed in [35]. It would be interesting to search for rigid supersymmetric backgrounds within this theory as well, with the goal of systematically constructing supersymmetric Lifshitz or Schrödinger field theories.

With explicit non-relativistic supersymmetric backgrounds now available, the next step to exploring the concept of non-relativistic supersymmetry further would be to explicitly construct Lagrangians. Following the ideas of rigid supersymmetry, one way to accomplish this is to consider realizations of matter multiplets in Newton-Cartan supergravity and freeze out gravity to obtain a non-relativistic SUSY algebra. Knowledge of the transformation rules then allows one to build supersymmetric Lagrangians systematically [25, 40].

The study of relativistic supersymmetric field theories has recently led to a plethora of new results and a deeper understanding of strongly coupled field theories and holography. Further developing the concepts of non-relativistic supersymmetry and supergravity may turn out to be equally fruitful, and may provide us with valuable tools to study nonrelativistic field theories and gauge/gravity dualities.

\section{Acknowledgments}

We would like to thank Anthony Charles, Daniel Mayerson and Leopoldo Pando Zayas for interesting discussions. This work was supported in part by the US Department of Energy under grant DE-SC0007859.

\section{A Notation and conventions}

We choose the $2+1$ dimensional Dirac matrices to be

$$
\gamma^{A}=\left\{i \sigma^{2}, \sigma^{1}, \sigma^{3}\right\},
$$

where $\sigma^{i}$ are Pauli matrices, and $A=0,1,2$ denote flat tangent space indices. The Dirac matrices satisfy the following duality relations:

$$
\begin{aligned}
\gamma_{A B} & =-\epsilon_{A B C} \gamma^{C}, \\
\gamma_{A B C} & =-\epsilon_{A B C} .
\end{aligned}
$$

Here $\epsilon_{A B C}$ is the Levi-Civita symbol, with $\epsilon_{012}=1$. These identities imply the useful relations

$$
\begin{gathered}
\gamma_{a b}=\epsilon_{a b} \gamma_{0}, \\
\gamma_{a 0}=\epsilon_{a b} \gamma_{b},
\end{gathered}
$$

where $a, b=1,2$ and $\epsilon_{12}=1$. 
Note that when using curved indices, $\epsilon$ needs to be replaced by the Levi-Civita tensor $\omega$, so that for example $\gamma_{\mu \nu \rho}=-\omega_{\mu \nu \rho}$. The Levi-Civita tensor is related to the $\epsilon$-symbol by

$$
\omega_{\mu \nu \rho}=\sqrt{-g} \epsilon_{\mu \nu \rho}
$$

We can define a charge conjugation matrix $C=\gamma^{0}$, with the following properties:

$$
\begin{aligned}
C^{T} & =C^{-1}=-C=-C^{*} \\
C \gamma^{A} C^{-1} & =-\left(\gamma^{A}\right)^{T}
\end{aligned}
$$

A Dirac spinor $\psi$ in $2+1$ dimensions consists of two complex components. We define the Dirac conjugate in the usual way as $\bar{\psi} \equiv \psi^{\dagger} \gamma^{0}$, and the charge conjugate as $\psi^{c} \equiv \psi^{T} C$. Majorana spinors satisfy the Majorana condition

$$
\bar{\psi}=\psi^{c},
$$

which implies that $\psi$ has two real components.

\section{B Bilinear analysis of $\mathcal{N}=2$ backgrounds}

In this appendix, we construct supersymmetric backgrounds preserving at least one supersymmetry by performing an invariant tensor analysis following the work of [39, 41]. We focus once again on the $\mathcal{N}=(2,0)$ theory. For a similar analysis in the $\mathcal{N}=(1,1)$ case, see [38].

The $D=3, \mathcal{N}=(2,0)$ superalgebra is specified by a pair of two component Majorana spinors $\eta^{i}[17]$. We take $\eta^{i}$ to be commuting, and form a complete set of bilinears

$$
\kappa^{[i j]}=\bar{\eta}^{i} \eta^{j}, \quad K_{\mu}^{(i j)}=\bar{\eta}^{i} \gamma_{\mu} \eta^{j} .
$$

We can equivalently write

$$
\kappa=\kappa^{12}, \quad K_{\mu}=\frac{1}{2}\left(K_{\mu}^{11}+K_{\mu}^{22}\right), \quad L_{\mu}^{1}=K_{\mu}^{12}, \quad L_{\mu}^{2}=\frac{1}{2}\left(K_{\mu}^{11}-K_{\mu}^{22}\right) .
$$

The set of bilinears comprises one scalar and three vectors, corresponding to ten components, as expected for the symmetric combination of four spinor components.

The bilinears are not all independent, but may be related via Fierz identities. The relevant ones are the norms of the vectors

$$
K_{\mu} K^{\mu}=-L_{\mu}^{1} L^{1 \mu}=-L_{\mu}^{2} L^{2 \mu}=-\kappa^{2},
$$

the outer product relation

$$
L_{\mu}^{1} L_{\nu}^{1}+L_{\mu}^{2} L_{\nu}^{2}=K_{\mu} K_{\nu}-\eta_{\mu \nu} K_{\lambda} K^{\lambda},
$$

and the identities

$$
\epsilon_{\mu}^{\nu \rho} L_{\nu}^{1} L_{\rho}^{2}=\kappa K_{\mu}, \quad \epsilon_{\mu}^{\nu \rho} L_{\nu}^{2} K_{\rho}=-\kappa L_{\mu}^{1}, \quad \epsilon_{\mu}^{\nu \rho} K_{\nu} L_{\rho}^{1}=-\kappa L_{\mu}^{2},
$$

demonstrating that the vectors form a basis for the three-dimensional spacetime. 
We now turn to the differential identities that may be obtained from the Killing spinor equation (3.1)

$$
0=\delta \Psi_{\mu i}=\nabla_{\mu} \eta_{i}+\epsilon^{i j} \eta_{j} V_{\mu}-\gamma_{\mu} \eta_{i} D+\frac{1}{4} \gamma_{\mu} \gamma_{\rho \sigma} F^{\rho \sigma} \epsilon^{i j} \eta_{j}
$$

We find

$$
\begin{aligned}
\partial_{\mu} \kappa^{i j}= & \frac{1}{2} \varepsilon_{i j} F_{\mu \nu} K^{k k \nu} \\
\nabla_{\mu} K_{\nu}^{i j}= & 2 D \epsilon_{\mu \nu \lambda} K^{i j \lambda}-\frac{1}{2} F_{\mu \nu} \delta^{i j} \varepsilon^{k l} \kappa^{k l} \\
& +\left(\left(\frac{1}{4} \epsilon_{\mu \lambda \sigma} F^{\lambda \sigma}-V_{\mu}\right) \delta_{\nu}^{\rho}-\frac{1}{2} F_{\mu \sigma} \epsilon_{\nu}^{\rho \sigma}\right)\left(\varepsilon^{i k} K_{\rho}^{j k}+\varepsilon^{j k} K_{\rho}^{i k}\right) .
\end{aligned}
$$

Using (B.2), we have

$$
\begin{aligned}
\partial_{\mu} \kappa & =F_{\mu \nu} K^{\nu} \\
\nabla_{\mu} K_{\nu} & =2 D \epsilon_{\mu \nu \lambda} K^{\lambda}-F_{\mu \nu} \kappa \\
\nabla_{\mu} L_{\nu}^{a} & =2 D \epsilon_{\mu \nu}^{\lambda} L_{\lambda}^{a}-\epsilon^{a b} \epsilon_{\lambda \sigma[\mu} F^{\lambda \sigma} L_{\nu]}^{b}-\frac{1}{2} g_{\mu \nu} \epsilon^{a b} \epsilon^{\rho \lambda \sigma} F_{\lambda \sigma} L_{\rho}^{b}+2 \epsilon^{a b} V_{\mu} L_{\nu}^{b},
\end{aligned}
$$

or equivalently

$$
\begin{aligned}
d \kappa & =-i_{K} F \\
d K & =4 D * K-2 F \kappa \\
d L^{a} & =4 D * L^{a}+2 \epsilon^{a b} L^{b} \wedge * F+2 \epsilon^{a b} V \wedge L^{b},
\end{aligned}
$$

along with

$$
\begin{aligned}
& \nabla_{(\mu} K_{\nu)}=0 \\
& \nabla_{(\mu} L_{\nu)}^{a}=-\frac{1}{2} g_{\mu \nu} \epsilon^{a b} \epsilon^{\rho \lambda \sigma} F_{\lambda \sigma} L_{\rho}^{b}+2 \epsilon^{a b} V_{(\mu} L_{\nu)}^{b} .
\end{aligned}
$$

We can immediately see that $K^{\mu}$ is a Killing vector with norm given by $K_{\mu} K^{\mu}=-\kappa^{2}$. The analysis then proceeds in two cases: $K^{\mu}$ being timelike, and $K^{\mu}$ null.

\section{B.1 Timelike case}

If $\kappa \neq 0, K^{\mu}$ is a timelike Killing vector. We proceed by choosing adapted coordinates such that $K^{\mu} \partial_{\mu}=\partial / \partial t$ and writing the metric as

$$
d s^{2}=-\kappa^{2}(d t+\omega)^{2}+H^{2}\left(d x_{1}^{2}+d x_{2}^{2}\right)
$$

where the metric functions are $\kappa\left(x^{a}\right), \omega_{a}\left(x^{b}\right)$ and $H\left(x^{a}\right)$. Introduction of the natural dreibein basis

$$
e^{0}=\kappa(d t+\omega), \quad e^{a}=H d x^{a},
$$


allows us to write $K=-\kappa e^{0}=-\kappa^{2}(d t+\omega)$. Acting with the exterior derivative gives $d K=-2 d \kappa \wedge e^{0}-\kappa^{2} d \omega$. Comparison with (B.10) then allows us to solve for $F$

$$
F=-e^{0} \wedge \frac{d \kappa}{\kappa}-2 D e^{1} \wedge e^{2}+\frac{1}{2} \kappa d \omega .
$$

Note that the Bianchi identity $d F=0$ constrains $D\left(x^{a}\right)$ to be independent of $t$.

To proceed, we note that the algebraic identities imply that $L^{1}$ and $L^{2}$ span the 2dimensional space orthogonal to $e^{0}$. Hence we may write

$$
L^{a}=\kappa\left(\cos \psi \delta^{a b}+\sin \psi \epsilon^{a b}\right) e^{b},
$$

where $\psi\left(t, x^{a}\right)$ parametrizes a local frame rotation. Substitution of this expression for $L^{a}$ into the identity for $d L^{a}$ in (B.10) allows us to determine $V_{\mu}$

$$
V=\frac{1}{2}\left(d \psi-\left(*_{2} d \omega\right) e^{0}+*_{2} d \log (\kappa / H)\right)
$$

where $*_{2}$ is the Hodge dual on the 2-dimensional space spanned by $e^{1} \wedge e^{2}$. There is one remaining condition to check, which is the symmetrized $\nabla_{(\mu} L_{\nu)}^{a}$ differential identity in (B.11). However, explicit computation shows that this is automatically satisfied for the configuration above.

To summarize, supersymmetric backgrounds with a timelike Killing vector can be written as

$$
d s^{2}=-\kappa^{2}(d t+\omega)^{2}+H^{2}\left(d x_{1}^{2}+d x_{2}^{2}\right),
$$

along with the auxiliary fields

$$
\begin{aligned}
D & =D\left(x^{a}\right), \\
F=d M & =-e^{0} \wedge \frac{d \kappa}{\kappa}-2 D e^{1} \wedge e^{2}+\frac{1}{2} \kappa d \omega, \\
V & =\frac{1}{2}\left(d \psi-\left(*_{2} d \omega\right) e^{0}+*_{2} d \log (\kappa / H)\right) .
\end{aligned}
$$

The solution is specified by the arbitrary (but time-independent) functions $\kappa\left(x^{a}\right), \omega_{a}\left(x^{b}\right)$, $H\left(x^{a}\right)$ and $D\left(x^{a}\right)$. Note that the function $\psi\left(t, x^{a}\right)$ is a gauge parameter, and can be set to zero if desired.

Given this background field configuration, we can now return to the Killing spinor equation (B.6). After some manipulation, we find that the Killing spinors have the form

$$
\eta^{i}=\sqrt{\kappa}\left(\cos (\psi / 2)+\gamma_{0} \sin (\psi / 2)\right) \eta_{0}^{i}
$$

where $\eta_{0}^{i}$ satisfies the $\frac{1}{2}$-BPS projection

$$
\eta_{0}^{i}=\gamma_{0} \varepsilon_{i j} \eta_{0}^{j}
$$

Although the analysis proceeded by assuming only one unbroken supersymmetry out of four, we see that the background actually preserves at least two supersymmetries. 
While the background (B.18) is generically $\frac{1}{2}$-BPS, the supersymmetry can be completely unbroken for appropriate choices of the fields. Such backgrounds ought to match those obtained by the integrability analysis of section 5 . However, note that there is no $a$ priori reason that the choice of metric in (B.18) needs to coincide with the ones of section 5 . In fact, the dual field strength from (B.14)

$$
f=\left(2 D-\frac{1}{2} \kappa *_{2} d \omega\right) e^{0}+H^{-1} *_{2} d \log \kappa
$$

does not necessarily even point along a single adapted coordinate direction, and hence falls outside of the ansätze used in section 5. Of course, we expect the backgrounds to be related by appropriate coordinate transformations.

\section{B.2 Null case}

We now turn to the null case, corresponding to $\kappa=0$. Following [39], we note from (B.10) and (B.11) that $K^{\mu}$ satisfies $K \wedge d K=0$ and $K^{\mu} \nabla_{\mu} K^{\nu}=0$, so it is both hypersurface orthogonal and tangent to affinely parametrized geodesics. This allows us to introduce null coordinates $(u, v, x)$ and write

$$
K^{\mu} \frac{\partial}{\partial x^{\mu}}=\frac{\partial}{\partial v}, \quad K_{\mu} d x^{\mu}=H^{-1} d u .
$$

We then specialize the metric to take the form

$$
d s^{2}=H^{-1}\left(\mathcal{F} d u^{2}+2 d u d v\right)+e^{2 \sigma} d x^{2},
$$

where the functions $H(u, x), \mathcal{F}(u, x)$ and $\sigma(u, x)$ are independent of $v$. We use the dreibein basis

$$
e^{+}=H^{-1} d u, \quad e^{-}=d v+\frac{1}{2} \mathcal{F} d u, \quad e^{3}=e^{\sigma} d x,
$$

and take the tangent space metric to be $\eta_{+-}=\eta_{33}=1$.

When $\kappa=0$, the first identity in (B.10) places a constraint on $F$

$$
F=F_{+3}(u, x) e^{+} \wedge e^{3},
$$

where independence of $v$ arises from demanding the Bianchi identity $d F=0$. The second identity in (B.10) allows us to solve for $D$

$$
D=-\frac{1}{4} e^{-\sigma} \partial_{x} \log H
$$

Note, curiously, that this is similar to the expression (5.44) obtained from integrability in the null case, however with the opposite sign. Nevertheless, there is no inconsistency since the null Killing vectors (5.42) and (B.22) are distinct, so that the corresponding adapted metrics are not directly equivalent.

Given $F$ and $D$, what remains is to use the differential identities for $L^{a}$ in (B.10) and (B.11) to solve for $V$. In order to do so, we note that substituting $\kappa=0$ in (B.5) shows that $L^{a} \wedge K=0$, so that $L^{a}$ is parallel to $K$. This allows us to write $L^{a}=\phi^{a} K$ 
where $\phi^{a}(u, v, x)$ can in principle depend on all coordinates. The Fierz identity (B.4) then demonstrates that $\left(\phi^{a}\right)^{2}=1$, so that we can express

$$
L^{1}=K \cos \psi, \quad L^{2}=K \sin \psi
$$

in terms of a single function $\psi(u, v, x)$. We can now solve for $V$, and find the simple pure gauge result

$$
V=-\frac{1}{2} d \psi
$$

In summary, for the null Killing vector case, the supersymmetric background is given by

$$
d s^{2}=H^{-1}\left(\mathcal{F} d u^{2}+2 d u d v\right)+e^{2 \sigma} d x^{2},
$$

along with the auxiliary fields

$$
\begin{aligned}
D & =-\frac{1}{4} e^{-\sigma} \partial_{x} \log H, \\
F=d M & =F_{u x} d u \wedge d x, \\
V & =-\frac{1}{2} d \psi .
\end{aligned}
$$

This solution is specified by the metric functions $H(u, x), \mathcal{F}(u, x)$ and $\sigma(u, x)$ as well as by $F_{u x}(u, x)$. As in the timelike case, the function $\psi(u, v, x)$ is a gauge parameter, and can be set to zero.

Returning to the Killing spinor equation, we find that the Killing spinors have the form

$$
\eta=e^{\frac{i}{2} \psi \sigma^{2}} \eta_{0}, \quad \gamma^{1} \eta_{0}=0 .
$$

Here we have used a shorthand notation of combining the two spinor parameters $\left(\eta^{1}, \eta^{2}\right)$ into a two-component vector which is acted upon by the Pauli matrix $\sigma^{2}$. The projection $\gamma^{1} \eta_{0}=0$ demonstrates that this is generically a $\frac{1}{2}$-BPS background.

Open Access. This article is distributed under the terms of the Creative Commons Attribution License (CC-BY 4.0), which permits any use, distribution and reproduction in any medium, provided the original author(s) and source are credited.

\section{References}

[1] E. Bergshoeff, J. Rosseel and T. Zojer, Newton-Cartan (super)gravity as a non-relativistic limit, Class. Quant. Grav. 32 (2015) 205003 [arXiv: 1505.02095] [INSPIRE].

[2] D.T. Son, Newton-Cartan geometry and the quantum Hall effect, arXiv:1306.0638 [INSPIRE].

[3] M. Geracie, D.T. Son, C. Wu and S.-F. Wu, Spacetime symmetries of the quantum Hall effect, Phys. Rev. D 91 (2015) 045030 [arXiv:1407.1252] [INSPIRE].

[4] A. Gromov and A.G. Abanov, Thermal Hall effect and geometry with torsion, Phys. Rev. Lett. 114 (2015) 016802 [arXiv: 1407.2908] [INSPIRE]. 
[5] M.H. Christensen, J. Hartong, N.A. Obers and B. Rollier, Torsional Newton-Cartan geometry and Lifshitz holography, Phys. Rev. D 89 (2014) 061901 [arXiv:1311.4794] [InSPIRE].

[6] M.H. Christensen, J. Hartong, N.A. Obers and B. Rollier, Boundary stress-energy tensor and Newton-Cartan geometry in Lifshitz holography, JHEP 01 (2014) 057 [arXiv:1311.6471] [INSPIRE].

[7] J. Hartong, E. Kiritsis and N.A. Obers, Lifshitz space-times for Schrödinger holography, Phys. Lett. B 746 (2015) 318 [arXiv:1409.1519] [InSPIRE].

[8] R. Banerjee, A. Mitra and P. Mukherjee, Localization of the Galilean symmetry and dynamical realization of Newton-Cartan geometry, Class. Quant. Grav. 32 (2015) 045010 [arXiv: 1407.3617] [INSPIRE].

[9] J. Hartong, E. Kiritsis and N.A. Obers, Field theory on Newton-Cartan backgrounds and symmetries of the Lifshitz vacuum, JHEP 08 (2015) 006 [arXiv:1502.00228] [INSPIRE].

[10] K. Jensen, On the coupling of Galilean-invariant field theories to curved spacetime, arXiv: 1408.6855 [INSPIRE].

[11] M. Geracie, K. Prabhu and M.M. Roberts, Fields and fluids on curved non-relativistic spacetimes, JHEP 08 (2015) 042 [arXiv: 1503.02680] [INSPIRE].

[12] J.F. Fuini, A. Karch and C.F. Uhlemann, Spinor fields in general Newton-Cartan backgrounds, Phys. Rev. D 92 (2015) 125036 [arXiv:1510.03852] [INSPIRE].

[13] C.W. Misner, K.S. Thorne and J.A. Wheeler, Gravitation, W.H. Freeman, San Francisco U.S.A. (1973).

[14] G. Dautcourt, On the newtonian limit of general relativity, Acta Phys. Po. B 21 (1990) 755.

[15] R. Andringa, E. Bergshoeff, S. Panda and M. de Roo, Newtonian gravity and the Bargmann algebra, Class. Quant. Grav. 28 (2011) 105011 [arXiv:1011.1145] [INSPIRE].

[16] R. Andringa, E.A. Bergshoeff, J. Rosseel and E. Sezgin, 3D Newton-Cartan supergravity, Class. Quant. Grav. 30 (2013) 205005 [arXiv: 1305.6737] [InSPIRE].

[17] P.S. Howe, J.M. Izquierdo, G. Papadopoulos and P.K. Townsend, New supergravities with central charges and Killing spinors in (2+1)-dimensions, Nucl. Phys. B 467 (1996) 183 [hep-th/9505032] [INSPIRE].

[18] A. Achucarro and P.K. Townsend, Extended supergravities in $d=(2+1)$ as Chern-Simons theories, Phys. Lett. B 229 (1989) 383 [INSPIRE].

[19] J. Teschner, Exact results on $\mathcal{N}=2$ supersymmetric gauge theories, Math. Phys. Stud. 2016 (2016) 1 [arXiv: 1412.7145].

[20] V. Pestun, Localization of gauge theory on a four-sphere and supersymmetric Wilson loops, Commun. Math. Phys. 313 (2012) 71 [arXiv:0712.2824] [InSPIRE].

[21] N. Bobev, H. Elvang, D.Z. Freedman and S.S. Pufu, Holography for $N=2^{*}$ on $S^{4}$, JHEP 07 (2014) 001 [arXiv:1311.1508] [INSPIRE].

[22] M. Leblanc, G. Lozano and H. Min, Extended superconformal Galilean symmetry in Chern-Simons matter systems, Annals Phys. 219 (1992) 328 [hep-th/9206039] [INSPIRE].

[23] D. Tong and C. Turner, Quantum Hall effect in supersymmetric Chern-Simons theories, Phys. Rev. B 92 (2015) 235125 [arXiv: 1508.00580] [InSPIRE]. 
[24] N. Doroud, D. Tong and C. Turner, On superconformal anyons, JHEP 01 (2016) 138 [arXiv: 1511.01491] [INSPIRE].

[25] G. Festuccia and N. Seiberg, Rigid supersymmetric theories in curved superspace, JHEP 06 (2011) 114 [arXiv:1105.0689] [INSPIRE].

[26] B. Jia and E. Sharpe, Rigidly supersymmetric gauge theories on curved superspace, JHEP 04 (2012) 139 [arXiv:1109.5421] [INSPIRE].

[27] H. Samtleben and D. Tsimpis, Rigid supersymmetric theories in $4 D$ Riemannian space, JHEP 05 (2012) 132 [arXiv: 1203.3420] [INSPIRE].

[28] C. Klare, A. Tomasiello and A. Zaffaroni, Supersymmetry on curved spaces and holography, JHEP 08 (2012) 061 [arXiv: 1205.1062] [INSPIRE].

[29] T.T. Dumitrescu, G. Festuccia and N. Seiberg, Exploring curved superspace, JHEP 08 (2012) 141 [arXiv:1205.1115] [INSPIRE].

[30] J.T. Liu, L.A. Pando Zayas and D. Reichmann, Rigid supersymmetric backgrounds of minimal off-shell supergravity, JHEP 10 (2012) 034 [arXiv:1207.2785] [INSPIRE].

[31] T.T. Dumitrescu and G. Festuccia, Exploring curved superspace (II), JHEP 01 (2013) 072 [arXiv: 1209.5408] [INSPIRE].

[32] K. Jensen and A. Karch, Revisiting non-relativistic limits, JHEP 04 (2015) 155 [arXiv: 1412.2738] [INSPIRE].

[33] M. Roček and P. van Nieuwenhuizen, $N \geq 2$ supersymmetric Chern-Simons terms as $D=3$ extended conformal supergravity, Class. Quant. Grav. 3 (1986) 43 [INSPIRE].

[34] H. Nishino and S.J. Gates, Jr., Chern-Simons theories with supersymmetries in three-dimensions, Int. J. Mod. Phys. A 8 (1993) 3371 [INSPIRE].

[35] E. Bergshoeff, J. Rosseel and T. Zojer, Newton-Cartan supergravity with torsion and Schrödinger supergravity, JHEP 11 (2015) 180 [arXiv:1509.04527] [INSPIRE].

[36] C. Closset, T.T. Dumitrescu, G. Festuccia and Z. Komargodski, Supersymmetric field theories on three-manifolds, JHEP 05 (2013) 017 [arXiv:1212.3388] [INSPIRE].

[37] K. Hristov, A. Tomasiello and A. Zaffaroni, Supersymmetry on three-dimensional lorentzian curved spaces and black hole holography, JHEP 05 (2013) 057 [arXiv:1302.5228] [INSPIRE].

[38] N.S. Deger, A. Kaya, H. Samtleben and E. Sezgin, Supersymmetric warped AdS in extended topologically massive supergravity, Nucl. Phys. B 884 (2014) 106 [arXiv:1311.4583] [INSPIRE].

[39] J.P. Gauntlett, J.B. Gutowski, C.M. Hull, S. Pakis and H.S. Reall, All supersymmetric solutions of minimal supergravity in five- dimensions, Class. Quant. Grav. 20 (2003) 4587 [hep-th/0209114] [INSPIRE].

[40] G. Knodel, J.T. Liu and L.A. Pando Zayas, On $N=1$ partition functions without R-symmetry, JHEP 03 (2015) 132 [arXiv:1412.4804] [INSPIRE].

[41] K.P. Tod, All metrics admitting supercovariantly constant spinors, Phys. Lett. B 121 (1983) 241 [INSPIRE]. 